\title{
High-resolution FUSE and HST ultraviolet spectroscopy of the white dwarf central star of Sh 2-216
}

\author{
T. Rauch ${ }^{1}$, M. Ziegler ${ }^{1}$, K. Werner ${ }^{1}$, J. W. Kruk ${ }^{2}$, C. M. Oliveira ${ }^{2}$, D. Vande Putte ${ }^{3}$, R. P. Mignani ${ }^{3}$, and F. Kerber ${ }^{4}$ \\ ${ }^{1}$ Institut für Astronomie und Astrophysik, Universität Tübingen, Sand 1, 72076 Tübingen, Germany \\ e-mail: rauch@astro.uni-tuebingen.de \\ 2 Department of Physics and Astronomy, Johns Hopkins University, Baltimore, MD 21218, USA \\ 3 Mullard Space Science Laboratory, University College London, Holmbury St Mary, Dorking, Surrey RH5 6NT, UK \\ ${ }^{4}$ European Southern Observatory, Karl-Schwarzschild-Straße 2, 85748 Garching, Germany
}

Received 24 January 2007 / Accepted 11 April 2007

\section{ABSTRACT}

\begin{abstract}
Context. We perform a comprehensive spectral analysis of LS V $+46^{\circ} 21$ in order to compare its photospheric properties to theoretical predictions from stellar evolution theory as well as from diffusion calculations.

Aims. LS V $+46^{\circ} 21$ is the DAO-type central star of the planetary nebula Sh 2-216. High-resolution, high- $S / N$ ultraviolet observations obtained with FUSE and STIS aboard the HST as well as the optical spectrum have been analyzed in order to determine the photospheric parameters and the spectroscopic distance.

Methods. We performed a detailed spectral analysis of the ultraviolet and optical spectrum by means of state-of-the-art NLTE modelatmosphere techniques.

Results. From the N IV - N v, O IV - O VI, Si IV - Si v, and Fe v - Fe vII ionization equilibria, we determined an effective temperature of $(95 \pm 2) \mathrm{kK}$ with high precision. The surface gravity is $\log g=6.9 \pm 0.2$. An unexplained discrepancy appears between the spectroscopic distance $d=224_{-58}^{+46} \mathrm{pc}$ and the parallax distance $d=129_{-5}^{+6} \mathrm{pc}$ of LS V $+46^{\circ} 21$. For the first time, we have identified Mg IV and $\mathrm{Ar}$ VI absorption lines in the spectrum of a hydrogen-rich central star and determined the $\mathrm{Mg}$ and $\mathrm{Ar}$ abundances as well as the individual abundances of iron-group elements $(\mathrm{Cr}, \mathrm{Mn}, \mathrm{Fe}, \mathrm{Co}$, and $\mathrm{Ni})$. With the realistic treatment of metal opacities up to the iron group in the model-atmosphere calculations, the so-called Balmer-line problem (found in models that neglect metal-line blanketing) vanishes.

Conclusions. Spectral analysis by means of NLTE model atmospheres has presently arrived at a high level of sophistication, which is now hampered largely by the lack of reliable atomic data and accurate line-broadening tables. Strong efforts should be made to improve upon this situation.
\end{abstract}

Key words. ISM: planetary nebulae: individual: Sh 2-216 - stars: abundances - stars: atmospheres - stars: evolution stars: individual: LS V $+46^{\circ} 21$ - stars: AGB and post-AGB

\section{Introduction}

The planetary nebula (PN) Sh 2-216 (PN G158.6+00.7) has been discovered as a large and faint emission nebula (Sharpless 216) and was classified as an H II region. Fesen et al. (1981) performed spectrophotometry on this "curious emission-line nebula" and found several forbidden lines and properties similar to PNe. Reynolds (1985) used high-resolution Fabry-Perot spectrometry to show that Sh 2-216 has all the characteristics of an extremely old PN with a very low expansion velocity $v_{\exp }<4 \mathrm{~km} \mathrm{~s}^{-1}$, but a central star (CS) was not found.

* Based on observations with the NASA/ESA Hubble Space Telescope, obtained at the Space Telescope Science Institute, which is operated by the Association of Universities for Research in Astronomy, Inc., under NASA contract NAS5-26666.

$\star \star$ Based on observations made with the NASA-CNES-CSA Far Ultraviolet Spectroscopic Explorer. FUSE is operated for NASA by the Johns Hopkins University under NASA contract NAS5-32985.

$\star \star \star$ Table A.1 is only available in electronic form at the CDS via anonymous ftp to cdsarc.u-strasbg.fr (130.79.128.5) or via http://cdsweb.u-strasbg.fr/cgi-bin/qcat? J/A+A/470/317

Figures A.1 and A.2 are available in electronic form at http://www . aanda.org (they are also available at the CDS in FITS format).
Reynolds (1985) speculated that the CS is no longer centrally located due to deceleration of the PN shell over a long period of time by the interstellar medium (ISM).

Cudworth \& Reynolds (1985) have unambiguously identified LS V $+46^{\circ} 21$, one of two possible CS candidates (the other was AS 84, Hardorp et al. 1965) located nearly midway between the apparent center of Sh 2-216 and its eastern rim, as the exciting star of Sh 2-216 by proper-motion measurements. Sh 2-216 has obviously experienced a mild interaction with the ISM (Tweedy et al. 1995). From its distance and proper motion, Kerber et al. (2004) have determined that it has a thin-disk orbit of low inclination and eccentricity, and that the CS left the center of its surrounding PN about 45000 years ago.

At a distance of $d=129 \mathrm{pc}$ (Harris et al. 2007), it is the closest possible PN known. With an apparent size of $100^{\prime} \times 100^{\prime}$ (Borkowski et al. 1990), it is among the physically most extended (cf. Rauch et al. 2004) and hence, oldest PNe (about 660000 years, Napiwotzki 1999) known.

Since its identification as the CS of Sh 2-216, LS V +46 21 has been object of many investigations and analyses which are briefly summarized here.

Feibelman \& Bruhweiler (1990) were able to detect a large number of $\mathrm{Fe} v$ and $\mathrm{Fe}$ vI lines in spectra taken by the 
International Ultraviolet Explorer (IUE). Tweedy \& Napiwotzki (1992) have demonstrated that LS V $+46^{\circ} 21$, which is the brightest $\left(m_{\mathrm{V}}=12.67 \pm 0.02\right.$, Cheselka et al. 1993) DAO-type white dwarf (WD 0441+467, McCook \& Sion 1999) known, has the properties $\left(T_{\text {eff }}=90 \mathrm{kK}, \log g=7\left(\mathrm{~cm} \mathrm{~s}^{-2}\right), \mathrm{He} / \mathrm{H}=0.01\right.$ by number) necessary to ionize the surrounding nebula.

Napiwotzki (1992, 1993), Napiwotzki \& Schönberner (1993), and Napiwotzki \& Rauch (1994) have reported that the Balmer-series lines of very hot DAO white dwarfs could not all be fit simultaneously with an NLTE model of a given $T_{\text {eff }}$. For example, fits to the individual Balmer lines of $\mathrm{LS} \mathrm{V}+46^{\circ} 21$ gave values of $T_{\text {eff }}$ ranging from about $50 \mathrm{kK}$ for $\mathrm{H} \alpha$ to $90 \mathrm{kK}$ for $\mathrm{H} \delta$. This circumstance is known as the Balmer line problem (BLP). Bergeron et al. (1993) found (in LTE calculations for DAO WDs) that the BLP is reduced by the consideration of metal-line blanketing, and Bergeron et al. (1994) could show clearly that the presence of heavy metals is the source of the BLP. Barstow et al. (1998) have shown that models which neglect the opacity of heavy elements are not well suited for the analysis of DA white dwarfs. To summarize, pure hydrogen models are not suited for the spectral analysis of hot DA(O) WDs in general (cf. Fig. 12).

Werner (1996) calculated NLTE model atmospheres for LS V $+46^{\circ} 21$ based on parameters of Tweedy \& Napiwotzki (1992) and introduced C, N, and O (at solar abundances) in addition. Surface cooling by these metals as well as the detailed consideration of the Stark line broadening in the modelatmosphere calculation has the effect that the BLP almost vanishes in $\mathrm{LS} \mathrm{V}+46^{\circ} 21$. Later, Kruk \& Werner (1998) could demonstrate that these model atmospheres reproduce well HUT (Hopkins Ultraviolet Telescope) observations of LS V $+46^{\circ} 21$ within $912-1840 \AA$ at $T_{\text {eff }}=85 \mathrm{kK}$ and $\log g=6.9$.

Napiwotzki (1999) determined $T_{\text {eff }}=83.2 \pm 3.3 \mathrm{kK}$ and $\log g=6.74 \pm 0.19$. The model contained only $\mathrm{H}$ and $\mathrm{He}$ (at an abundance ratio of $\left.\log n_{\mathrm{He}} / n_{\mathrm{H}}=-1.95\right)$ and a fit to $\mathrm{H} \delta$ was done to derive $T_{\text {eff }}$ (cf. Fig. 12). Also, instead of assuming LTE, which fails in the region of typical white dwarf central stars $\left(T_{\text {eff }}=100 \mathrm{kK} / \log g=7.0\right)$, he calculated an NLTE model based on the accelerated lambda iteration (ALI) method as described by Werner (1986).

Werner et al. (2003a) evaluated the Fe v - Fe vi ionization equilibrium and arrived at $T_{\text {eff }}=90 \mathrm{kK}$ and $\log g=7.0$.

Traulsen et al. (2005) considered the opacity of light metals $(\mathrm{C}, \mathrm{N}, \mathrm{O}$, and $\mathrm{Si})$ in addition and determined $T_{\text {eff }}=93 \pm 5 \mathrm{kK}$ and $\log g=6.9 \pm 0.2\left(\mathrm{~cm} \mathrm{~s}^{-1}\right) . T_{\text {eff }}$ and $\log g$ were derived from the ionization equilibrium of $\mathrm{O}$ IV $-\mathrm{O}$ VI. The abundances for the included elements were determined to be $[\mathrm{He}]=-0.9,[\mathrm{C}]=-1.0$, $[\mathrm{N}]=-2.0,[\mathrm{O}]=-0.9$, and $[\mathrm{Si}]=-0.3([\mathrm{x}]: \log$ (mass fraction $/$ solar mass fraction) of species $\mathrm{x}$ ). From $\mathrm{O} v \lambda 1371 \AA$ a radial velocity of $v_{\text {rad }}=+22.4 \mathrm{~km} \mathrm{~s}^{-1}$ was measured.

Recently, Hoffmann (2005) determined an oversolar iron abundance in a preliminary analysis based on $\mathrm{H}+\mathrm{He}+\mathrm{Fe}$ models.

In this paper, we present a detailed analysis of the individual abundances of iron-group elements and light metals based on high-resolution UV observations (Sect. 2). The analysis is described in Sect. 3.

\section{Observations and reddening}

Spectral analysis by model-atmosphere techniques needs observations of lines of successive ionization stages in order to evaluate the ionization equilibrium of a particular species which is a sensitive indicator of $T_{\text {eff }}$. For stars with $T_{\text {eff }}$ as high as $\approx 90 \mathrm{kK}$, the ionization degree is very high and
Table 1. FUSE observations of LS V $+46^{\circ} 21$ used in this analysis.

\begin{tabular}{cccc}
\hline \hline Dataset & Start time (UT) & Aperture & Exp time (s) \\
\hline M1070404 & 2001-01-10 15:29 & LWRS & 5606 \\
M1070407 & 2001-01-25 14:46 & LWRS & 6826 \\
M1070416 & 2003-02-06 09:59 & LWRS & 7511 \\
M1070419 & 2003-09-27 00:48 & LWRS & 3541 \\
M1070422 & $2004-01-2317: 40$ & LWRS & 2990 \\
\hline M1070402 & $2001-01-0917: 36$ & MDRS & 1418 \\
M1070405 & $2001-01-2316: 15$ & MDRS & 8922 \\
M1070408 & $2001-01-2519: 58$ & MDRS & 6257 \\
M1070417 & $2003-02-0615: 05$ & MDRS & 4899 \\
M1070420 & $2003-09-2715: 55$ & MDRS & 3613 \\
M1070423 & $2004-01-2606: 49$ & MDRS & 4401 \\
\hline
\end{tabular}

most of the metal lines are found at UV wavelengths. Thus, high- $S / N$ and high-resolution UV spectra are a prerequisite for a precise analysis. Consequently, we used FUSE (Far Ultraviolet Spectroscopic Explorer) and HST/STIS (Space Telescope Imaging Spectrograph aboard the Hubble Space Telescope) in order to obtain suitable data.

\subsection{The FUSE spectrum of $L S V+46^{\circ} 21$}

FUSE provides spectra in the wavelength band 900-1187 $\AA$ with a typical resolving power of 20000 . FUSE consists of four independent co-aligned telescopes with prime-focus Rowland circle spectrographs. Two of the four channels have optics coated with $\mathrm{Al}+\mathrm{LiF}$ and two channels have optics coated with $\mathrm{SiC}$. Each spectrograph has three entrance apertures: LWRS $\left(30^{\prime \prime} \times 30^{\prime \prime}\right)$, MDRS $\left(4^{\prime \prime} \times 20^{\prime \prime}\right)$, and HIRS $\left(1^{\prime \prime} .25 \times 20^{\prime \prime}\right)$. Further information on the FUSE mission and instrument can be found in Moos et al. (2000) and Sahnow et al. (2000).

This star was observed many times in all three spectrograph apertures (LWRS, MDRS, and HIRS) as part of the wavelength calibration program for FUSE. The observations used in this analysis are listed in Table 1 . The LWRS observations were photometric or nearly so in all four channels, so the effective exposure time was essentially the same for each of them. The effective exposure time varied considerably in the MDRS channels as a result of channel misalignments. The time given in Table 1 for these observations is that of the LiF1 channel; the LiF2 time is similar but the $\mathrm{SiC}$ channel exposure time is typically lower by a factor of two.

The raw data for each exposure were reduced using CalFUSE version 3.0.7. For a description of the CalFUSE pipeline, see Dixon et al. (2007). The exposure with the maximum flux outside of airglow lines was then identified for each observation. Any exposures with less than $40 \%$ of this flux were discarded and the remainder were normalized to match the peak exposure and their exposure times were scaled accordingly. These corrections were negligible (less than 1\%) for all but the last exposure of M1070422, where a delayed target acquisition resulted in a significant correction. For the MDRS spectra these corrections were negligible for the LiF channels but were typically $\sim 50 \%$ for the SiC channels. The MDRS spectra were subsequently renormalized to match the LWRS spectra. The exposures were coaligned by cross-correlating on narrow ISM features and combined. This coalignment was then repeated to combine the observations; the end result was a single spectrum for each channel and spectrograph aperture. Subsequent analyses were performed primarily using the LWRS spectra, with the MDRS spectra providing a confirmation that weak features were real and not the result of detector fixed-pattern noise. 
At first glance, the FUSE spectrum of LS V $+46^{\circ} 21$ gives the impression that it is hopeless to find signatures of the stellar photosphere in the sea of interstellar absorption lines (Fig. 1). However, some strong, isolated lines, e.g. of $\mathrm{N}$ IV, P v, S vi, and $\mathrm{Cr}$ VI, as well as a large number of Fe VI, Fe VII, and Ni VI lines, are identified and are included in our analysis.

In order to demonstrate that a combined model of photospheric and interstellar absorption reproduces the FUSE observation well, we decided to apply the profile-fitting procedure OWENS which enables us to model the interstellar absorption lines with high accuracy. The use of this procedure is described in Sect. 4.

\subsection{The STIS spectrum of $L S V+46^{\circ} 21$}

The STIS observation had been performed with the E140M grating and the FUV-MAMA detector which provides échelle spectra in the wavelength range from $1144 \AA$ to $1729 \AA$ with a theoretical resolving power of $\lambda / \Delta \lambda=45800$ ). Two spectra (total exposure time $5.5 \mathrm{ks}$, resolution $\approx 0.06 \AA$ ) were taken in 2000 and processed by the standard pipeline data reduction (version of June 2006).

In order to increase the signal-to-noise ratio $(S / N)$, the two obtained spectra have been co-added and were subsequently smoothed with a Savitzky-Golay filter (Savitzky \& Golay 1964). This spectrum has a very $\operatorname{good} S / N>50$.

\subsection{Interstellar absorption and reddening}

In order to fine tune model-atmosphere parameters and better match the observations, we have determined from all models both the column density of interstellar neutral hydrogen $\left(n_{\mathrm{HI}}\right)$ and the reddening $\left(E_{B-V}\right)$.

From a detailed comparison of $\mathrm{H}_{\mathrm{I}} \mathrm{L} \alpha$ (Fig. 1) with the observation (best suited because the interstellar $\mathrm{H}$ I absorption dominates the complete line profile), we determined $N_{\mathrm{HI}}=$ $(8.5 \pm 1.0) \times 10^{19} \mathrm{~cm}^{-2}$. For the following analysis, we adopt this value.

We determine the reddening from the FUSE and STIS spectra (Fig. 2). We note that the FUSE and STIS fluxes agree quite well for $1150-1180 \AA$. We achieve the best match to the continuum slope with $E_{B-V}=0.065_{-0.015}^{+0.010}$ (Fig. 2)

This value is higher than the $E_{B-V}=0.024 \pm 0.008$ predicted by the Galactic reddening law of Groenewegen \& Lamers (1989). In the case of LS V $+46^{\circ} 21$ its PN is likely to modify the expect reddening behaviour in two ways: first, the enormous ambient PN will result in additional reddening (although the column density is small), and second, presence of the circumstellar matter is probably modifying the applied reddening law (Seaton 1979). Our $E_{B-V}$ is lower than $E_{B-V}=0.1$ that Kruk \& Werner (1998) derived from the analysis of a HUT spectrum (915 $<\lambda<1840 \AA$ ). Recently, Harris et al. (2007) have determined $E_{B-V}=0.08$ from optical spectra.

We have to mention that fitting the extinction is uncertain. The generic curves are averages to many sight lines and often don't fit a single one very well. The curves are less well-characterized as one goes farther into the FUV, as there are multiple absorbers possible and they each have a distinctive wavelength-dependence (see, e.g., Sofia et al. 2005). However, the exact $E_{B-V}$ is not important, neither for our following spectral analysis by detailed line-profile fitting (Sect. 3.3) nor for our distance determination (Sect. 3.4). We finally adopt $E_{B-V}=0.065$.

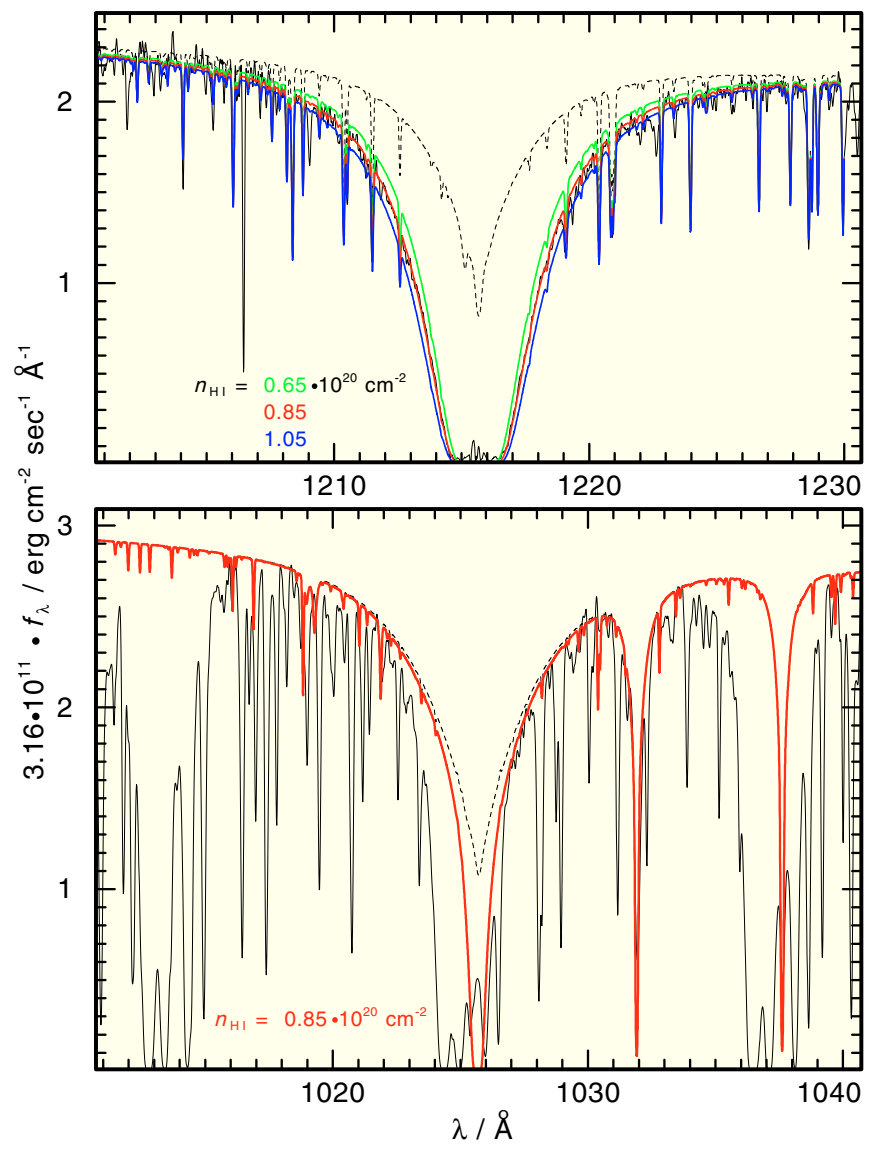

Fig. 1. Synthetic spectra around H I Ly $\alpha$ (top) and Ly $\beta$ (bottom) calculated with different $n_{\mathrm{HI}}$ compared with sections of the STIS and FUSE spectra of LS V $+46^{\circ} 21$, respectively. Note the strong interstellar absorption features in the lower panel. An additional factor of 0.85 is applied in order to normalize the synthetic stellar spectrum to the observation at $1020 \AA$ and $1030 \AA$. The blue wing of $\operatorname{Ly} \beta$ is blended with interstellar $\mathrm{H}_{2}(J=0,1)$ absorption. The observation of $\mathrm{Ly} \alpha$ is best matched at $n_{\mathrm{HI}}=8.5 \times 10^{19} \mathrm{~cm}^{-2}$. The thin, dashed spectra are calculated without interstellar $\mathrm{H}$ I absorption. For the model-atmosphere parameters, see Sect.7. All synthetic spectra shown in this paper are normalized to match the observation at $1700 \AA$ and are convolved with Gaussians in order to match the instrument resolution (Sect. 2). The observed spectrum is normalized by a factor of $3.16 \times 10^{11}$.

\section{Data modeling and analysis}

In the following, we describe in detail the analysis of the FUSE and STIS spectra of LS V $+46^{\circ} 21$ by means of NLTE modelatmosphere techniques.

\subsection{Model atmospheres and atomic data}

We employed TMAP, the Tübingen NLTE Model Atmosphere Package (Werner et al. 2003b), for the calculation of planeparallel, homogeneous, static models which consist of $\mathrm{H}, \mathrm{He}, \mathrm{C}$, $\mathrm{N}, \mathrm{O}, \mathrm{F}, \mathrm{Mg}, \mathrm{Si}, \mathrm{P}, \mathrm{S}, \mathrm{Ar}, \mathrm{Ca}, \mathrm{Sc}, \mathrm{Ti}, \mathrm{V}, \mathrm{Cr}, \mathrm{Mn}, \mathrm{Fe}, \mathrm{Co}$, and $\mathrm{Ni}$ (in the following, we will refer to $\mathrm{Ca}-\mathrm{Ni}$ as iron-group elements). $\mathrm{H}, \mathrm{He}, \mathrm{C}, \mathrm{N}, \mathrm{O}, \mathrm{F}, \mathrm{Mg}, \mathrm{Si}, \mathrm{P}, \mathrm{S}$, and Ar are represented by "classical" model atoms (Rauch 1997) partly taken from TMAD, the Tübingen Model Atom Database ${ }^{1}$.

For $\mathrm{Ca}+\mathrm{Sc}+\mathrm{Ti}+\mathrm{V}+\mathrm{Cr}+\mathrm{Mn}+\mathrm{Fe}+\mathrm{Co}+\mathrm{Ni}$ individual model atoms are constructed by IrOnIc (Rauch \& Deetjen 2003),

\footnotetext{
${ }^{1}$ http://astro. uni-tuebingen.de/ rauch/TMAD/TMAD.html
} 


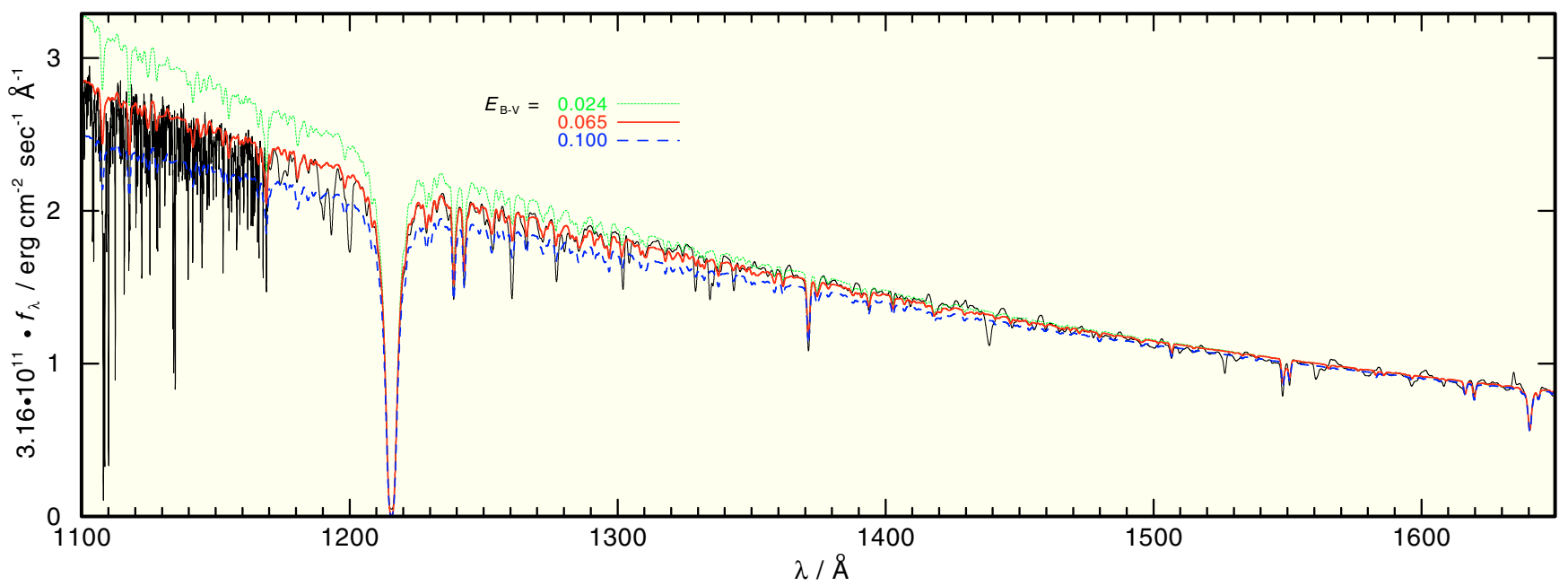

Fig. 2. Sections of the FUSE (1150-1170 $)$ ) and STIS (1170-1650 $)$ spectra of LS V $+46^{\circ} 21$ compared with synthetic spectra at different $E_{B-V}$ (for clarity, the FUSE and STIS spectra are smoothed with Gaussians of $0.1 \AA$ and $1 \AA F W H M$, respectively). The synthetic spectra are smoothed with Gaussians of $1 \AA F W H M$ and are normalized to the flux level of the observation around He II $\lambda 1640 \AA$. Due to the many (photospheric and interstellar) absorption lines, a convolution of the FUSE spectrum with a wider Gaussian would result in an artificially lower "continuum". The overall continuum is well reproduced at $E_{B-V}=0.065$.

using a statistical approach in order to treat the extremely large number of atomic levels and line transitions by the introduction of "super-levels" and "super-lines". In total 686 levels are treated in NLTE, combined with 2417 individual lines and about 9 million iron-group lines (Table 2), taken from Kurucz (1996) as well as from the OPACITY and IRON projects (Seaton et al. 1994; Hummer et al. 1993). It is worthwhile to mention that this is the most extended model we have calculated with $T M A D$ so far. The frequency grid comprises about 34000 points spanning $10^{12}-3 \times 10^{17} \mathrm{~Hz}$. On a PC of our institute's cluster with a $3.4 \mathrm{GHz}$ processor and $4 \mathrm{~GB}$ RAM, one iteration takes about $48000 \mathrm{~s}$. We need more than one week of CPU time to calculate one complete model with a convergence criterion of $10^{-4}$ in the relative corrections of temperature, densities, occupation numbers, and flux for all (90) depth points.

\subsection{Line identification}

The UV spectrum of $\mathrm{LS} \mathrm{V}+46^{\circ} 21$ exhibits a large number of stellar and interstellar absorption features. None of the identified photospheric lines shows evidence for on-going mass loss of LS V $+46^{\circ} 21$.

We are able to identify and reproduce about $95 \%$ of all spectral lines in the FUSE and STIS spectra of LS V $+46^{\circ} 21$. It is likely that most of the remaining unidentified lines (e.g. in Fig. 12) stem from the same ions, but are from transitions whose wavelengths are not sufficiently well-known. For instance for Fe VII, Kurucz (1996) provides only 22 laboratory measured (POS) lines and 1952 lines with theoretical line positions (LIN lines). This situation is even worse for other ions (Fe VI: 224 and 58664, respectively) and species.

Despite these problems, we identify weak Mg IV lines in the STIS spectrum (Table 3). The strongest of which, Mg IV $\lambda 1683.0 \AA$, is shown in Fig. 3. Noerdlinger \& Dynan (1975) had already proposed this identification for a respective feature in the supergiant $\delta$ Ori.

Moreover, we could identify Si IV and Si v lines (Table 3), which provide a new ionization equilibrium to evaluate for the $T_{\text {eff }}$ determination. An example for a $\mathrm{Si} v$ line is shown in Fig. 3.
We have also identified Ar vi $\lambda 1303.86 \AA$ as an isolated line in the STIS spectrum (Table 3) of LS V $+46^{\circ} 21$ (Fig. 4). To our best knowledge, this is the first time that Ar VI has been detected in the photosphere of any star.

Recently, Werner et al. (2005) have identified F v $\lambda \lambda 1082.31,1087.82,1088.39 \AA \quad$ and $\quad F$ vi $\lambda 1139.50 \AA$ in FUSE observations of hot central stars of planetary nebulae (CSPN). We have inspected the spectrum of LS V $+46^{\circ} 21$ but we cannot identify these lines unambiguously (Fig. 5). However, while a $10 \times$ solar $\mathrm{F}$ abundance is definitely too much, these $\mathrm{F}$ lines can be hidden in the spectrum at a solar $\mathrm{F}$ abundance. This is fully consistent with the result of Werner et al. (2005) that H-rich CSPN have approximately solar F abundances. For our calculations, we adopt a solar $\mathrm{F}$ abundance.

In addition, lines of $\mathrm{H}, \mathrm{He}, \mathrm{C}, \mathrm{N}, \mathrm{O}, \mathrm{P}, \mathrm{S}, \mathrm{Cr}, \mathrm{Mn}, \mathrm{Fe}, \mathrm{Co}$, and $\mathrm{Ni}$ have been identified as well.

The complete FUSE and STIS spectra compared to the synthetic spectra calculated from our final model with identification marks as well as a table with the wavelengths of all identified lines are available online. In Figs. 1, 5-8, 13, and 16, we show some details of the FUSE spectrum. In Figs. 1, 3, 4, 8-10, 12, and 13, we show some details of the STIS spectrum. These figures show representative samples of absorption by various photospheric constituents; discussion of the individual species is given in subsequent sections.

The observed spectra have been shifted to the rest wavelength of the photospheric lines.

\subsection{Analysis}

In the first part of this analysis, we will mainly concentrate on the STIS spectrum in which we find many isolated lines of many species and their ions. Since the number of species is large, it is impossible to calculate extended model-atmosphere grids on a reasonable time scale. Therefore, we pursued the following strategy. We start our analysis with model atmospheres based on parameters (Sect. 1) of Traulsen et al. (2005). We include Mg and $\mathrm{Ca}-\mathrm{Ni}$ in addition (solar abundance ratios). In a first step, we will then re-adjust $T_{\text {eff }}$ precisely and check $\log g$ (Sect. 3.3.3). Subsequently, we will fine-tune the $\mathrm{C}, \mathrm{N}$, and $\mathrm{O}$ abundances in 
Table 2. Statistics of "classical" (left) and iron-group $(\mathrm{Ca}-\mathrm{Ni}$, right) model atoms used in our calculations. We give the number of levels treated in NLTE and the respective line transitions. For the iron-group levels, we list the number of so-called "super levels" and sampled lines that are statistically combined to "super lines".

\begin{tabular}{|c|c|c|c|c|c|c|}
\hline Ion & Levels & Lines & Ion & $\begin{array}{l}\text { Super } \\
\text { Levels }\end{array}$ & $\begin{array}{l}\text { Super } \\
\text { Lines }\end{array}$ & $\begin{array}{r}\text { Sample } \\
\text { Lines }\end{array}$ \\
\hline $\mathrm{H} \mathrm{I}$ & 10 & 45 & $\mathrm{CaV}$ & 3 & 6 & 141956 \\
\hline H II & 1 & - & $\mathrm{Ca}$ VI & 7 & 24 & 114545 \\
\hline $\mathrm{He} \mathrm{I}$ & 5 & 3 & Ca VII & 7 & 27 & 71608 \\
\hline $\mathrm{He}$ II & 14 & 90 & Ca VIII & 1 & 0 & 0 \\
\hline He III & 1 & - & $\mathrm{Sc} \mathrm{V}$ & 3 & 6 & 65994 \\
\hline C III & 10 & 12 & Sc VI & 7 & 24 & 237271 \\
\hline C IV & 54 & 295 & Sc VII & 7 & 26 & 176143 \\
\hline $\mathrm{C} \mathrm{V}$ & 1 & 0 & Sc VIII & 1 & 0 & 0 \\
\hline $\mathrm{N}$ IV & 9 & 10 & $\mathrm{Ti} \mathrm{V}$ & 3 & 5 & 26654 \\
\hline $\mathrm{N} \mathrm{V}$ & 54 & 297 & Ti VI & 7 & 24 & 95448 \\
\hline $\mathrm{N}$ VI & 1 & 0 & Ti VII & 7 & 26 & 230618 \\
\hline O IV & 11 & 19 & Ti VIII & 1 & 0 & 0 \\
\hline $\mathrm{OV}$ & 90 & 608 & V v & 3 & 5 & 2123 \\
\hline $\mathrm{O}$ VI & 54 & 291 & V VI & 7 & 24 & 35251 \\
\hline O VII & 1 & 0 & V VII & 7 & 24 & 112883 \\
\hline $\mathrm{F} \mathrm{V}_{\mathrm{V}}$ & 8 & 9 & V VIII & 1 & 0 & 0 \\
\hline F VI & 6 & 4 & $\mathrm{Cr} \mathrm{v}$ & 3 & 5 & 43860 \\
\hline F VII & 2 & 1 & Cr VI & 7 & 24 & 4406 \\
\hline F VIII & 1 & 0 & Cr VII & 7 & 24 & 37070 \\
\hline Mg III & 1 & 0 & Cr VIII & 1 & 0 & 0 \\
\hline Mg IV & 8 & 7 & Mn V & 3 & 5 & 285376 \\
\hline $\mathrm{Mg} \mathrm{V}$ & 5 & 2 & Mn VI & 7 & 24 & 70116 \\
\hline Mg VI & 1 & 0 & Mn VII & 7 & 24 & 8277 \\
\hline Si III & 6 & 4 & In VIII & 1 & 0 & 0 \\
\hline Si IV & 16 & 44 & $\mathrm{Fe} \mathrm{V}$ & 3 & 5 & 793718 \\
\hline Si v & 15 & 20 & $\mathrm{Fe}$ VI & 7 & 25 & 340132 \\
\hline $\mathrm{Si} \mathrm{VI}$ & 1 & 0 & $\mathrm{Fe}$ VII & 7 & 24 & 86504 \\
\hline P III & 3 & 0 & $\mathrm{Fe}$ VIII & 1 & 0 & 0 \\
\hline P IV & 15 & 9 & $\mathrm{Co} \mathrm{V}$ & 3 & 5 & 1469717 \\
\hline $\mathrm{P}$ V & 18 & 12 & Co VI & 7 & 22 & 898484 \\
\hline P VI & 1 & 0 & Co VII & 7 & 23 & 492913 \\
\hline S IV & 6 & 4 & Co VIII & 1 & 0 & 0 \\
\hline $\mathrm{S} \mathrm{V}$ & 14 & 16 & $\mathrm{Ni} \mathrm{V}$ & 3 & 5 & 1006189 \\
\hline S VI & 18 & 48 & $\mathrm{Ni}$ VI & 7 & 22 & 1110584 \\
\hline S VII & 1 & 0 & Ni VII & 7 & 22 & 688355 \\
\hline Ar IV & 2 & 0 & Ni VIII & 1 & 0 & 0 \\
\hline Ar V & 9 & 6 & & & & \\
\hline Ar VI & 1 & 21 & & & & \\
\hline Ar VII & 2 & 36 & & & & \\
\hline Ar VIII & 13 & 24 & & & & \\
\hline Ar IX & 1 & 0 & & & & \\
\hline total & 522 & 1937 & & 162 & 480 & 8646195 \\
\hline
\end{tabular}

order to improve the fit to the STIS observation (Sect. 3.3.1). The abundances of $\mathrm{Mg}, \mathrm{Si}, \mathrm{P}, \mathrm{S}, \mathrm{Ar}$, and $\mathrm{Ca}-\mathrm{Ni}$ are then fine-tuned. During this whole process, our results for $T_{\text {eff }}$ and $\log g$ are continuously checked for consistency.

\subsubsection{Metal abundances}

The abundances of $\mathrm{C}, \mathrm{N}, \mathrm{O}, \mathrm{Mg}$, and Si have been determined by detailed line-profile fits based on the FUSE and STIS observations. Examples are shown in Figs. 3, 6, and 13.

In the FUSE spectrum of $\mathrm{LS} \mathrm{V}+46^{\circ} 21, \mathrm{P} \vee \lambda \lambda 1117,1128 \AA$ are identified (Fig. 7). These lines are well reproduced at a $0.5 \times$ solar abundance.

We have identified $S$ vi $\lambda \lambda 933,944 \AA$ in the FUSE and S VI $\lambda \lambda 1419.38,1419.74,1423.85 \AA$ in the STIS spectra (Fig. 8). The line cores of the $S$ vi resonance doublet appear too deep
Table 3. Identified $\mathrm{Mg}, \mathrm{Si}$, and Ar lines in the STIS spectrum of LS V $+46^{\circ} 21$.

\begin{tabular}{|c|c|c|c|c|}
\hline Ion & $\lambda / \AA$ & & Transition & \\
\hline $\mathrm{Mg}$ IV & 1336.850 & $3 p$ & ${ }^{4} \mathrm{P}_{5 / 2}^{\mathrm{o}}-3 \mathrm{~d}$ & ${ }^{4} \mathrm{D}_{3 / 2}$ \\
\hline $\mathrm{Mg}$ IV & 1342.163 & $3 p$ & ${ }^{4} \mathrm{P}_{5 / 2}^{\mathrm{o}}-3 \mathrm{~d}$ & ${ }^{4} \mathrm{D}_{5 / 2}$ \\
\hline $\mathrm{Mg}$ IV & 1346.543 & $3 p$ & ${ }^{4} \mathrm{P}_{5 / 2}^{\mathrm{o}}-3 \mathrm{~d}$ & ${ }^{4} \mathrm{D}_{7 / 2}$ \\
\hline $\mathrm{Mg}$ IV & 1352.020 & $3 p$ & ${ }^{4} \mathrm{P}_{3 / 2}^{\mathrm{o}}-3 \mathrm{~d}$ & ${ }^{4} \mathrm{D}_{5 / 2}$ \\
\hline $\mathrm{Mg}$ IV & 1387.494 & $3 p$ & ${ }^{4} \mathrm{D}_{5 / 2}^{\mathrm{o}}-3 \mathrm{~d}$ & ${ }^{4} \mathrm{~F}_{7 / 2}$ \\
\hline Mg IV & 1437.610 & $3 p$ & ${ }^{2} \mathrm{D}_{5 / 2}^{\mathrm{o}}-3 \mathrm{~d}$ & ${ }^{2} \mathrm{~F}_{7 / 2}$ \\
\hline Mg IV & 1490.433 & $3 \mathrm{~s}$ & ${ }^{4} \mathrm{P}_{3 / 2}-3 \mathrm{p}$ & ${ }^{4} \mathrm{~S}_{3 / 2}^{\mathrm{o}}$ \\
\hline Mg IV & 1520.967 & $3 p$ & ${ }^{4} S_{3 / 2}^{o}-3 d$ & ${ }^{4} \mathrm{P}_{1 / 2}^{3 / 2}$ \\
\hline Mg IV & 1658.851 & $3 \mathrm{~s}$ & ${ }^{4} \mathrm{P}_{5 / 2}^{3 / 2}-3 p$ & ${ }^{4} \mathrm{D}_{51}^{1 / 2}$ \\
\hline $\mathrm{Mg}$ IV & 1669.574 & $3 \mathrm{~s}$ & ${ }^{4} \mathrm{P}_{3 / 2}-3 \mathrm{p}$ & ${ }^{4} \mathrm{D}_{1 / 2}^{\mathrm{o} / 2}$ \\
\hline Mg IV & 1679.960 & $3 \mathrm{~s}$ & ${ }^{4} \mathrm{P}_{3 / 2}-3 \mathrm{p}$ & ${ }^{4} \mathrm{D}_{3 / 2}^{\mathrm{o}}$ \\
\hline $\mathrm{Mg}$ IV & 1683.003 & $3 \mathrm{~s}$ & ${ }^{4} \mathrm{P}_{5 / 2}^{3 / 2}-3 \mathrm{p}$ & ${ }^{4} \mathrm{D}_{7 / 2}^{\mathrm{o} / 2}$ \\
\hline Mg IV & 1692.675 & $3 \mathrm{~s}$ & ${ }^{4} \mathrm{P}_{1 / 2}^{5 / 2}-3 \mathrm{p}$ & ${ }^{4} \mathrm{D}_{1 / 2}^{\mathrm{o}}$ \\
\hline $\mathrm{Mg}$ IV & 1698.784 & $3 \mathrm{~s}$ & ${ }^{4} \mathrm{P}_{3 / 2}-3 \mathrm{p}$ & ${ }^{4} \mathrm{D}_{5 / 2}^{\mathrm{l} / 2}$ \\
\hline $\mathrm{Mg}$ IV & 1703.357 & $3 \mathrm{~s}$ & ${ }^{4} \mathrm{P}_{1 / 2}^{3 / 2}-3 \mathrm{p}$ & ${ }^{4} \mathrm{D}_{3 / 2}^{\mathrm{o}}$ \\
\hline Si IV & 1210.652 & $4 s$ & ${ }^{2} S_{1 / 2}-5 p$ & ${ }^{2} \mathrm{P}_{3 / 2}^{\mathrm{o}}$ \\
\hline Si IV & 1211.757 & $4 s$ & ${ }^{2} S_{1 / 2}-5 p$ & ${ }^{2} \mathrm{P}_{1 / 2}^{\mathrm{O} / 2}$ \\
\hline Si IV & 1402.770 & $3 \mathrm{~s}$ & ${ }^{2} S_{1 / 2}-3 p$ & ${ }^{2} \mathrm{P}_{1 / 2}^{\mathrm{o}}$ \\
\hline Si IV & 1533.219 & $4 d$ & ${ }^{2} \mathrm{D}_{5 / 2}-6 \mathrm{f}$ & ${ }^{2} \mathrm{~F}_{7 / 2}^{\mathrm{o}}$ \\
\hline Si IV & 1533.219 & $4 d$ & ${ }^{2} \mathrm{D}_{5 / 2}-6 \mathrm{f}$ & ${ }^{2} \mathrm{~F}_{5 / 2}^{\mathrm{o}}$ \\
\hline Si IV & 1533.222 & $4 d$ & ${ }^{2} \mathrm{D}_{3 / 2}-6 \mathrm{f}$ & ${ }^{2} \mathrm{~F}_{5 / 2}^{\mathrm{o}}$ \\
\hline Si IV & 1722.526 & $3 d$ & ${ }^{2} \mathrm{D}_{5 / 2}-4 \mathrm{p}$ & ${ }^{2} \mathrm{P}_{3 / 2}^{\mathrm{O}}$ \\
\hline Si IV & 1722.562 & $3 d$ & ${ }^{2} \mathrm{D}_{3 / 2}-4 \mathrm{p}$ & ${ }^{2} \mathrm{P}_{3 / 2}^{\mathrm{O}}$ \\
\hline Si IV & 1727.376 & $3 d$ & ${ }^{2} \mathrm{D}_{3 / 2}^{3 / 2}-4 \mathrm{p}$ & ${ }^{2} \mathrm{P}_{1 / 2}^{\mathrm{o} / 2}$ \\
\hline $\mathrm{Si} \mathrm{v}$ & 1235.453 & $3 \mathrm{~s}$ & ${ }^{3} \mathrm{P}_{2}^{\mathrm{o}}-3 \mathrm{p}$ & ${ }^{3} \mathrm{D}_{2}$ \\
\hline Si V & 1251.390 & $3 \mathrm{~s}$ & ${ }^{3} \mathrm{P}_{2}^{\mathrm{o}}-3 \mathrm{p}$ & ${ }^{3} \mathrm{D}_{3}$ \\
\hline Si V & 1276.008 & $3 \mathrm{~s}$ & ${ }^{3} \mathrm{P}_{1}^{\mathrm{o}}-3 \mathrm{p}$ & ${ }^{3} \mathrm{D}_{2}$ \\
\hline Si V & 1285.458 & $3 \mathrm{~s}$ & ${ }^{3} \mathrm{P}_{0}^{\mathrm{o}}-3 \mathrm{p}$ & ${ }^{3} \mathrm{D}_{1}$ \\
\hline $\mathrm{Si} \mathrm{V}$ & 1319.600 & $3 \mathrm{~s}$ & ${ }^{1} \mathrm{P}_{1}^{\mathrm{o}}-3 \mathrm{p}$ & ${ }^{1} \mathrm{D}_{2}$ \\
\hline Ar VI & 1283.934 & $3 p^{2}$ & ${ }^{2} \mathrm{P}_{1 / 2}-3 \mathrm{p}^{3}$ & ${ }^{2} \mathrm{D}_{3 / 2}^{\mathrm{o}}$ \\
\hline Ar vI & 1303.864 & $3 p^{2}$ & ${ }^{2} \mathrm{P}_{3 / 2}-3 \mathrm{p}^{3}$ & ${ }^{2} \mathrm{D}_{3 / 2}^{\mathrm{o}}$ \\
\hline Ar VI & 1307.349 & $3 p^{2}$ & ${ }^{2} \mathrm{P}_{3 / 2}-3 \mathrm{p}^{3}$ & ${ }^{2} \mathrm{D}_{5 / 2}^{\mathrm{o}}$ \\
\hline
\end{tabular}

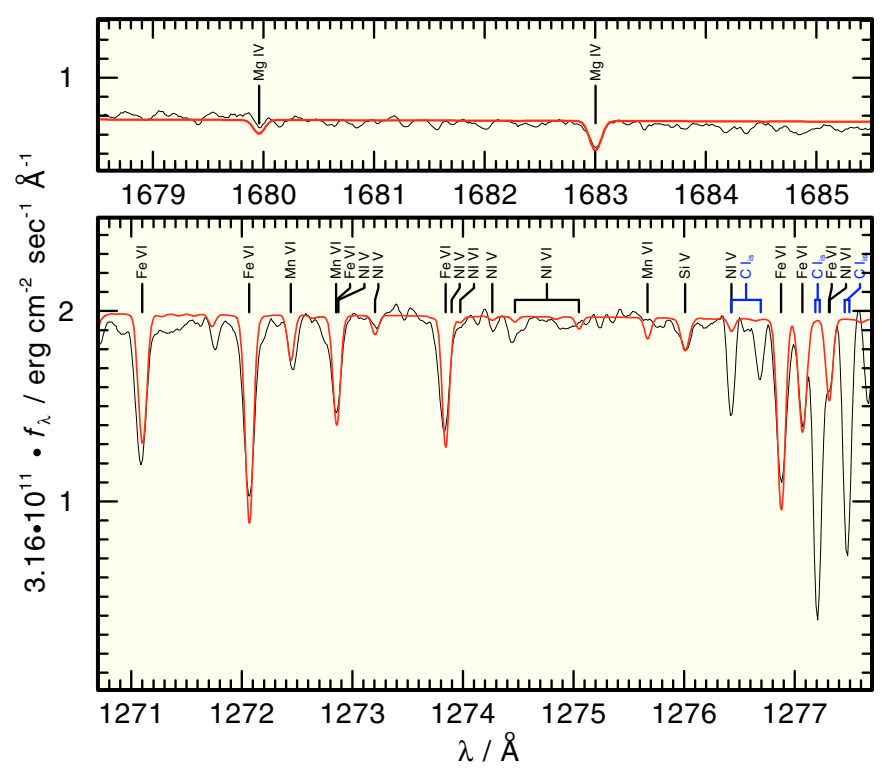

Fig. 3. Sections of the STIS spectrum of LSV $+46^{\circ} 21$ around Mg IV $\lambda 1683.0 \AA$ (top) and Si v $\lambda 1276.0 \AA$ (bottom). Identified lines are marked with their ion's name. "is" denotes interstellar lines. 


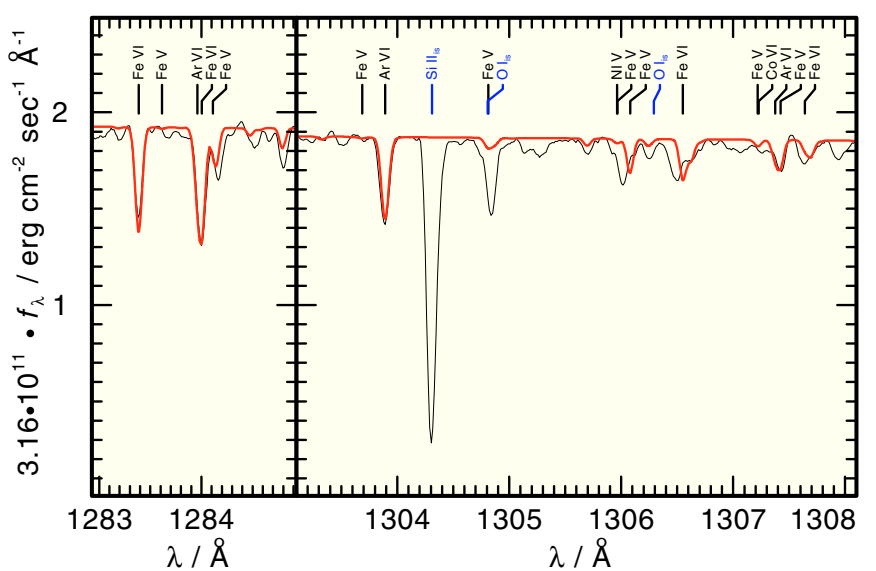

Fig. 4. Sections of the STIS spectrum of LS V $+46^{\circ} 21$ around Ar VI $\lambda \lambda$ 1283.93, 1303.86, $1307.35 \AA$.

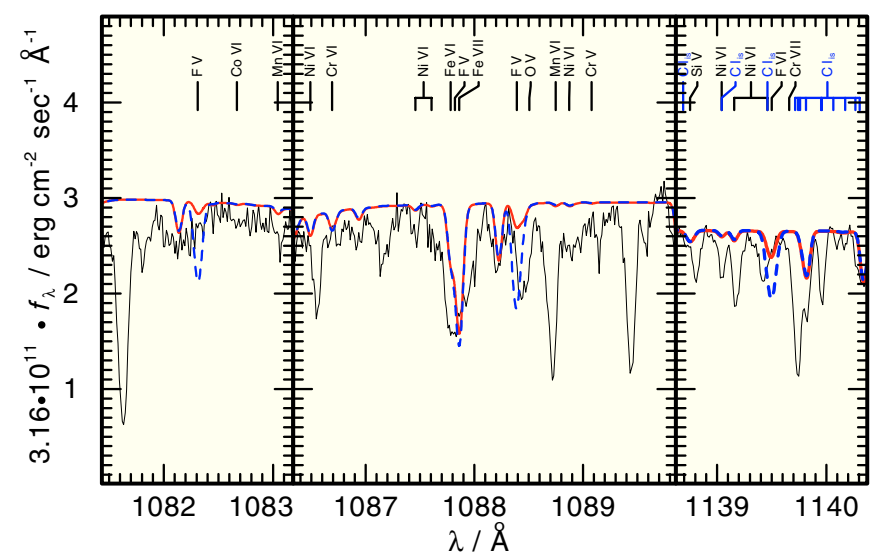

Fig. 5. Sections of the FUSE spectrum of LS V $+46^{\circ} 21$ around F V $\lambda \lambda 1082.31,1087.82,1088.39 \AA$ and F VI $\lambda 1139.50 \AA$ compared with synthetic spectra with solar (full line) and 10× solar F abundance (dashed line).

to match the observation and are not well-suited for an abundance determination. The reason might be the uncertain continuum level in the FUSE wavelength range due to the strong interstellar absorption and the unsufficient line-broadening tables. Though we use data of Dimitrijević \& Sahal-Bréchot (1993), these need to be extrapolated to the temperatures and densities at the line-forming regions especially of the line cores (which form in the outer parts of the atmosphere) and are, thus, not very accurate.

However, S vi $\lambda \lambda$ 1419.38, 1419.74, 1423.85 $($ Fig. 8) are well reproduced at a $0.5 \times$ solar abundance. $S$ vi $\lambda 1117.76 \AA$ (Fig. 7) is part of a strong blend but is in reasonable agreement with the observation. $\mathrm{S} \vee \lambda \lambda 1122,1129,1134 \AA$ as well as $S$ VI $\lambda \lambda 976,1000,1018 \AA$ are not identified but these are too strong at solar abundance.

We find discrepancies similar to those with the S VI resonance doublet's line cores (too deep) also for O VI (Fig. 13) where we use tables provided by Dimitrijević \& Sahal-Bréchot (1992a). We encounter the same problems in the STIS wavelength region where the continuum is much better defined with the resonance doublet of $\mathrm{N} v$ (Fig. 9, tables by Dimitrijević \& Sahal-Bréchot 1992b). In the case of C IV (Fig.9, tables by Dimitrijević et al. 1991), the absorption in the model is too weak; a strong interstellar absorption component may be the explanation.

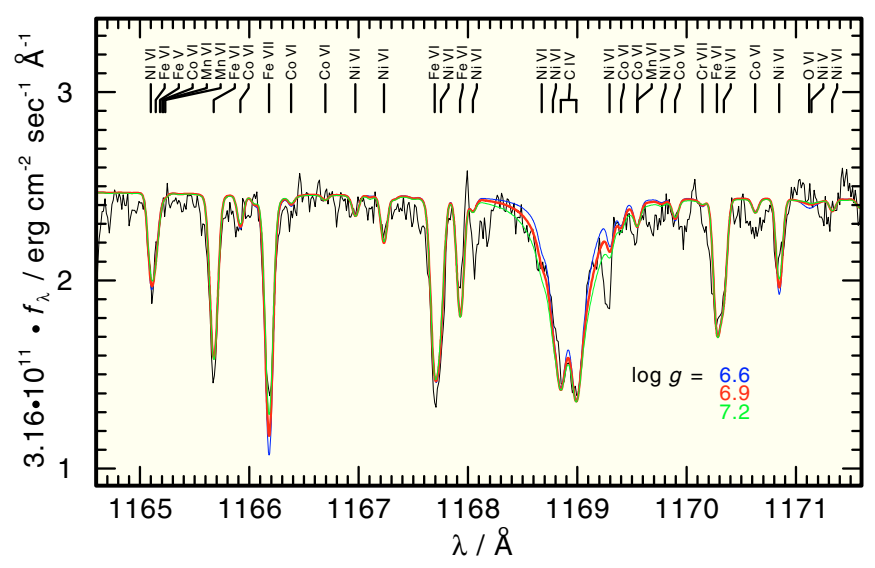

Fig. 6. Sections of the FUSE spectrum of LS V $+46^{\circ} 21$ around C IV $\lambda \lambda 1168.85,1168.99 \AA$ compared with synthetic spectra calculated from models with different $\log g$. While at $\log g=6.6$ the line wings of $\mathrm{C}$ IV $\lambda \lambda 1168.85,1168.99 \AA$ are too narrow, they appear too broad at $\log g=7.2$. The observation is best matched at $\log g=6.9$.

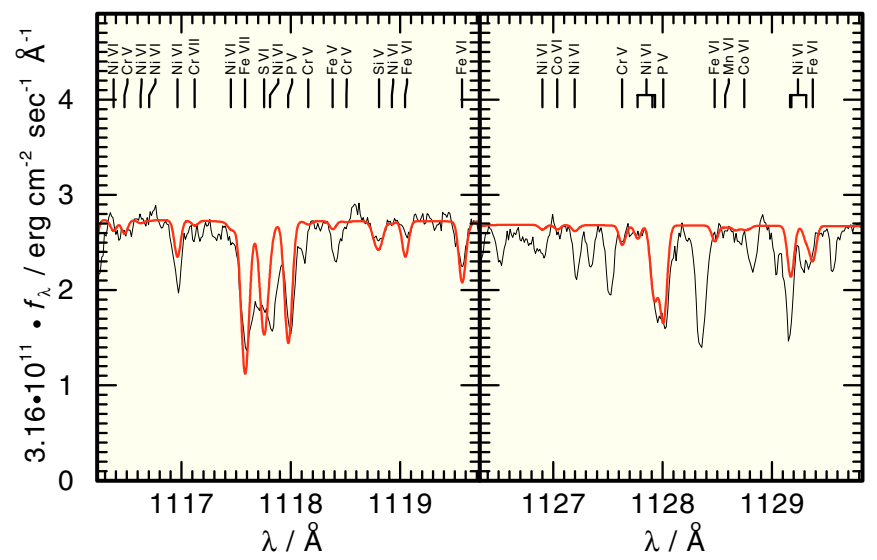

Fig. 7. Sections of the FUSE spectrum of LSV $+46^{\circ} 21$ around the P V $\lambda \lambda 1118.0,1128.1 \AA$ resonance doublet compared with our final model. Several lines in these sections are not identified.

In the STIS spectrum of $\mathrm{LS} \mathrm{V}+46^{\circ} 21$, we could identify iron-group lines of $\mathrm{Cr}, \mathrm{Mn}, \mathrm{Fe}, \mathrm{Co}$, and $\mathrm{Ni}$. For these we determined the abundances (Fig. 18) with an accuracy of 0.3 dex from line profile fits. No trace of $\mathrm{Ca}, \mathrm{V}, \mathrm{Sc}$, and Ti was found, neither in the STIS nor in the FUSE spectrum. We did some test calculations in order to find upper abundance limits, i.e. at what abundances do lines of these species appear too strongly to be in hidden in the observation. These limits are about 20-50 times solar. For our model calculations, however, we assume that the $\mathrm{Ca}, \mathrm{V}, \mathrm{Sc}$, and Ti have a solar-relative abundance pattern with the other iron-group elements and adopt a $5 \times$ solar abundance for them.

\subsubsection{Surface gravity}

Traulsen et al. (2005) calculated a spectroscopic distance of $d=$ $240 \pm 36$ pc (same method as described in Sect. 3.4). This is not in agreement with the result of Harris et al. (2007) who found $d=$ $128.9_{-5.3}^{+5.7} \mathrm{pc}$ from the trigonometric parallax. The spectroscopic distance is strongly dependent on $\log g$ (see Eq. (2)) which is determined from detailed line profile fits. From our experience, we know that in the relevant $\log g$ range (around 7), the typical error range is about $0.5 \mathrm{dex}$ - resulting in a large error in $d$. Since we have a direct measurement of the distance (Harris et al. 2007), 


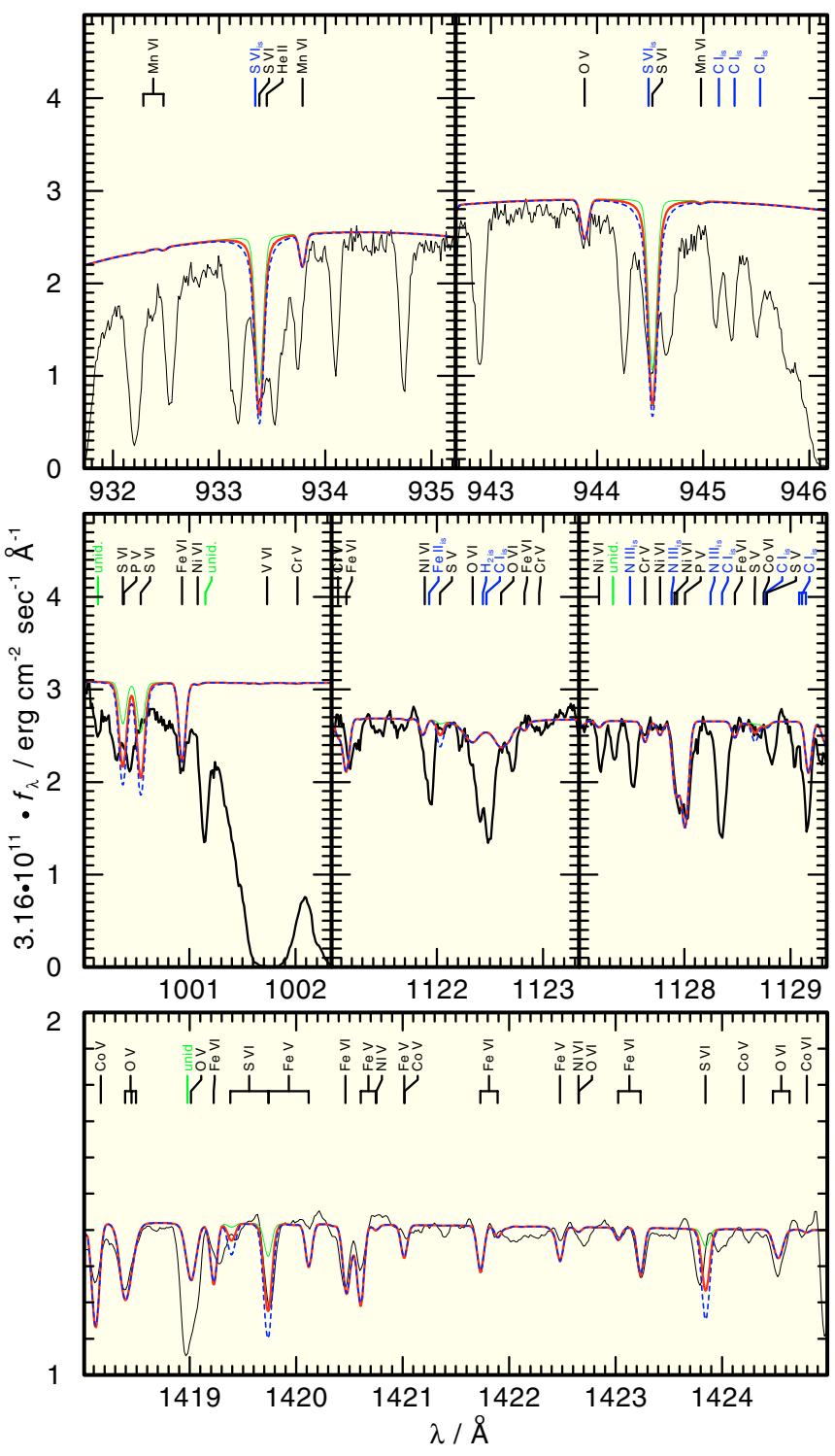

Fig. 8. Sections of the FUSE and STIS spectra of LS V $+46^{\circ} 21$ around S VI $\lambda \lambda 933.4,944.5 \AA$ (top), S v $\lambda \lambda 1122,1129 \AA$ and $\mathrm{S}$ VI $\lambda 1000 \AA$ (middle), and S VI $\lambda \lambda 1419.38,1419.74,1423.85 \AA$ (bottom). The synthetic spectra are calculated from models with solar (dashed line), $0.5 \times$ solar (thick line) and $0.1 \times$ solar (thin line) sulfur abundance. The strong unmarked absorption features in the top panel stem from interstellar $\mathrm{H}$ I and $\mathrm{H}_{2}$.

this adds a strong constraint (Sect. 3.4). From Eq. (2), we can estimate that we need a higher $\log g$ (of about 7.4) than found in previous analyses and thus, we extend our model-atmosphere grid to higher $\log g$ and higher $T_{\text {eff }}$. However, we find that a higher $\log g$ cannot be compensated by a change of $T_{\text {eff }}$ within the error limits derived from fits to the hydrogen Balmer lines. A detailed comparison of the He II $\lambda 1640 \AA 2-3$ multiplet with the STIS observation is shown in Fig. 10. He II $\lambda 1640 \AA$ is sensitive to both the He abundance as well as $\log g$. E.g. a factor of $0.5 / 2.0$ in the He abundance can be approximately compensated by $+0.5 /-0.5$ in $\log g$. However, from this line we derive $\log g=6.9 \pm 0.3$ and $[\mathrm{He}]=0.1 \pm 0.3$ dex. At $\log g=6.9$, e.g. the $\mathrm{C}$ IV $\lambda 1169 \AA$ A doublet is well reproduced (Fig. 6).

The determination of $\log g$ is a crucial issue of this work and thus, we tried to use the hydrogen Lyman lines for this purpose. Unfortunately, neither $\mathrm{L} \alpha$ which is in the STIS

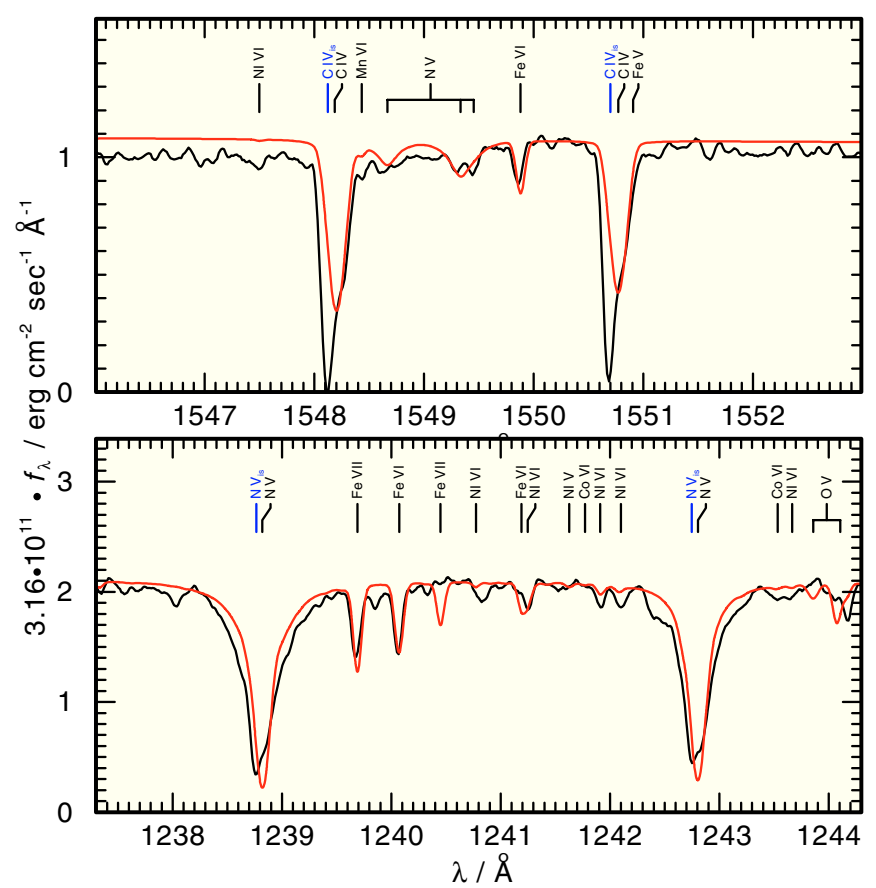

Fig. 9. Sections of the STIS spectrum of LSV $+46^{\circ} 21$ around C IV $\lambda \lambda 1548.2,1550.8 \AA$ and $N$ v $\lambda \lambda 1238.8,1242.8 \AA$ compared with our final model. The Fe VII $\lambda 1240.4 \AA$ line is obviously too strong, probably due to an error ( $f$-value too high) in Kurucz's line list (Kurucz 1996).

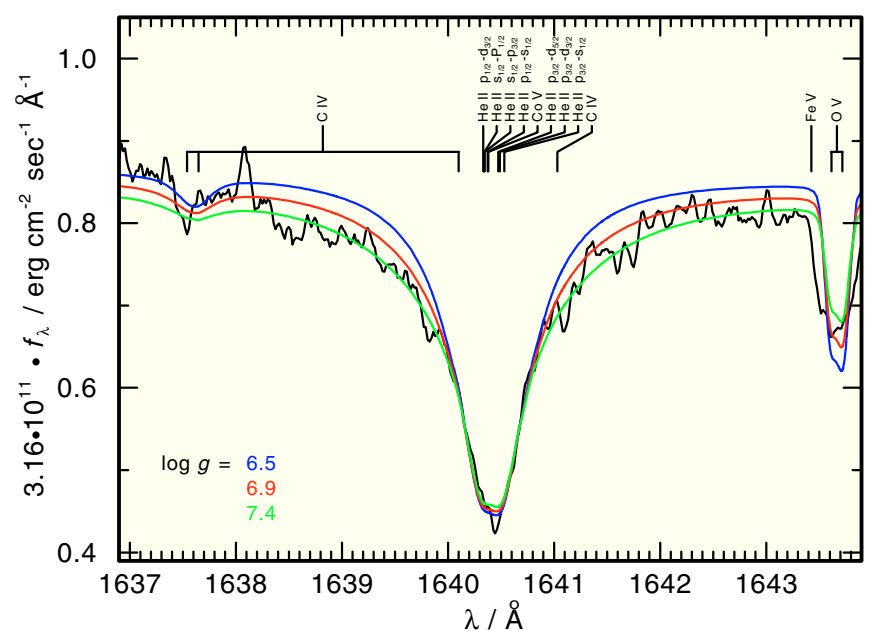

Fig. 10. Section of the STIS spectrum of LS V $+46^{\circ} 21$ around He II $\lambda \lambda 1640.32-1640.53 \AA$ compared with theoretical line profiles calculated from models with $T_{\text {eff }}=95 \mathrm{kK}$ and $\log g=6.5,6.9,7.3$. Note that the width of the fine-structure splitting amounts to $\approx 0.22 \AA$ and thus, has to be considered at STIS's resolution here. For the He II line broadening, we use tables by Schöning \& Butler (1989).

wavelength range nor the higher lines of the Lyman series are suited: while the complete $\mathrm{L} \alpha$ line profile is dominated by absorption of interstellar H I (Fig. 1), the strong interstellar absorption in the FUSE wavelength range at $\lambda \lesssim 1100 \AA$ strongly hampers, e.g., the determination of the local continuum. Thus, we have a look at the hydrogen Balmer lines because their line broadening is well known and detailed $\mathrm{H}$ I line-broadening tables are available (Lemke 1997). In Fig. 11 we show $\mathrm{H} \beta$ as an example. The determination of $\log g=6.9 \pm 0.2$ from the $\mathrm{UV}$ wavelength range is confirmed within even narrower error 


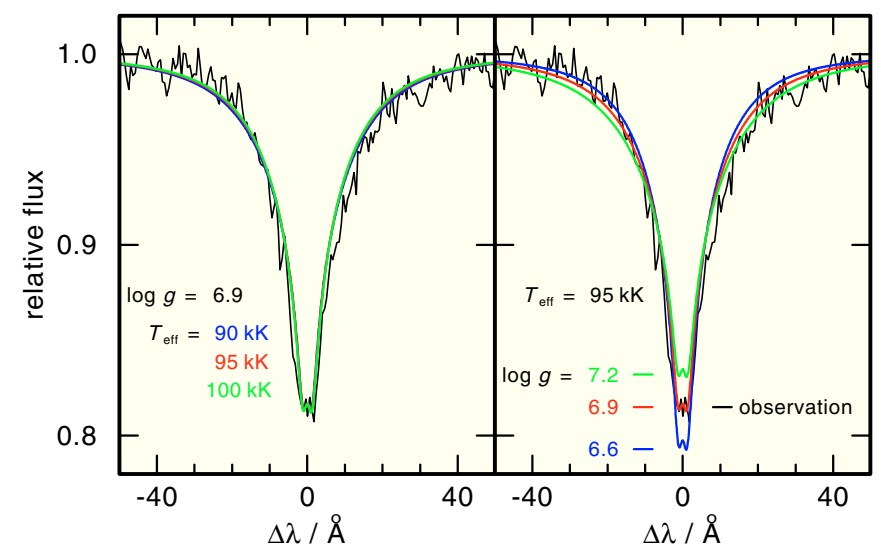

Fig. 11. Dependency of $\mathrm{H} \beta$ on $T_{\text {eff }}$ (left) and $\log g$ (right). A section of the optical spectrum of LS V $+46^{\circ} 21$ (taken in Oct. 1990 with the TWIN spectrograph attached to the $3.5 \mathrm{~m}$ telescope at the Calar Alto observatory) around $\mathrm{H} \beta$ compared with theoretical line profiles calculated from models with $T_{\text {eff }}=90,95,100 \mathrm{kK}$ and $\log g=6.5,6.9,7.3$. In this parameter range, $\mathrm{H} \beta$ appears not very sensitive on $T_{\text {eff }}$ but very sensitive on $\log g$ in the line center. At $\log g=6.9$, the central depression is perfectly matched and the observed line profile is well reproduced.

limits. Plots of the model spectrum computed with our final adopted parameters are shown with the observed Balmer line profiles in Fig. 20.

\subsubsection{Effective temperature}

An ionization equilibrium is a very sensitive indicator for $T_{\mathrm{eff}}$. The evaluation of ionization equilibria of many species with many lines increases the accuracy (e.g. Rauch 1993) further. As a prerequisite, lines of successive ions of a species have to be identified. In the STIS spectrum of LS V $+46^{\circ} 21$, we found lines of $\mathrm{N}$ IV $-\mathrm{N}$ v, O Iv $-\mathrm{O}$ v, $\mathrm{Si}$ IV $-\mathrm{Si}$ v, Fe v - Fe vII, and Ni v $\mathrm{Ni}$ VII which are suitable for this purpose. In addition, the FUSE spectrum provides lines of $\mathrm{N}$ IV, O VI, Si IV, Fe VI - Fe VII, and $\mathrm{Ni}$ VI - Ni VII.

With our synthetic spectra, we are able to reproduce all $\mathrm{O}$ lines but $\mathrm{O} \vee \lambda 1371 \AA$. The reason is unknown, however, a possible reason is the approximate formula used for the quadratic Stark effect. Thus, detailed line-broadening data for this line is highly desirable.

In Fig. 12 we show the dependency of the Fe v - Fe vII equilibrium on $T_{\text {eff. }}$. We can model Fe v - Fe vII lines simultaneously at $T_{\text {eff }}=95 \mathrm{kK}$. Figure 13 demonstrates that other ionization equilibria are also well reproduced at this $T_{\text {eff }}$. For other species, the reader may have a look at the complete FUSE and STIS spectra (Sect. 3.2).

Additional constraints for $T_{\text {eff }}$ are found in the optical wavelength range, where the observed $\mathrm{H} \alpha$ and $\mathrm{He}$ II $\lambda 4686 \AA$ line profiles (Figs. 20,14) show emission reversals in their line cores. These are reproduced by our models. Such emission cores can be used as a measure for $T_{\text {eff }}$ as well (Rauch et al. 1996). While the $\mathrm{H} \alpha$ emission core changes only little in the relevant $T_{\text {eff }}$ range, significant changes are visible in He II $\lambda 4686 \AA$ (Fig. 14). From this line, $T_{\text {eff }}=97 \mathrm{kK}$ can be determined. However, precise $T_{\text {eff }}$ determinations from emission reversal profiles require higher spectral resolution than the $1.5 \AA$ achieved in the present spectrum.

We finally adopt $T_{\text {eff }}=95 \mathrm{kK}$. From the comparison of synthetic spectra from models within $T_{\text {eff }}=90-100 \mathrm{kK}$, we estimate an error range in $T_{\text {eff }}$ of $2 \mathrm{kK}$.

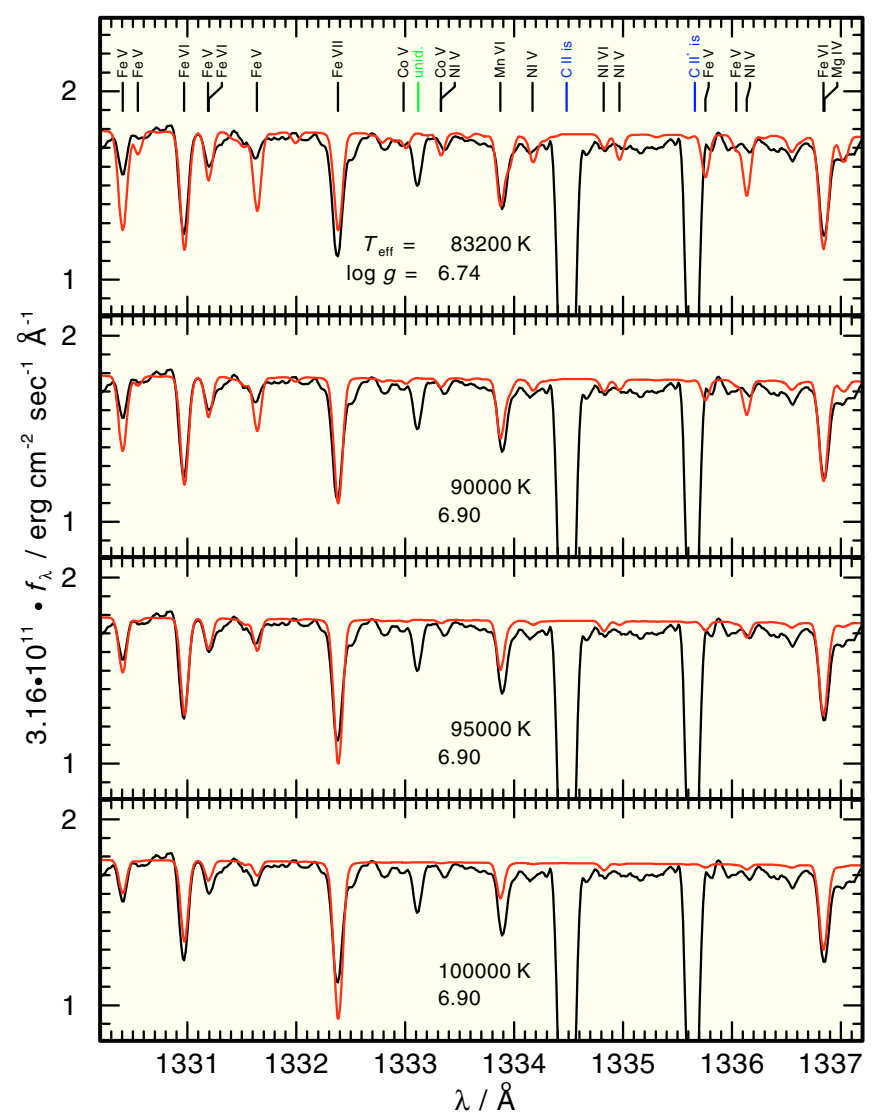

Fig. 12. Section of the STIS spectrum of LS V $+46^{\circ} 21$ compared to models with different $T_{\text {eff }}$ and $\log g$. The Fe V and Fe VII lines appear strongly dependent on changes of $T_{\text {eff }}$ while Fe VI is almost independent in the relevant $T_{\text {eff }}$ range. Fe V, Fe VI, and Fe VII are reproduced simultaneously at $T_{\text {eff }}=95 \mathrm{kK}$. Note that the $T_{\text {eff }}$ determination by Napiwotzki (1999) based on $\mathrm{H}+\mathrm{He}$ composed models resulted in a much too low $T_{\text {eff }}$ (top panel). "unid." denotes a unidentified line.

\subsection{Mass, distance, and luminosity}

In Fig. 15 we compare the position of LS V $+46^{\circ} 21$ to evolutionary tracks in the $\log T_{\text {eff }}-\log g$ plane. We can interpolate a mass of $M=0.550_{-0.015}^{+0.020} M_{\odot}$ and a luminosity of $\log L / L_{\odot}=2.2 \pm 0.2$ from the evolutionary tracks of Schönberner (1983) and Blöcker \& Schönberner (1990).

The spectroscopic distance of $\mathrm{LS} \mathrm{V}+46^{\circ} 21$ is calculated following the flux calibration of Heber et al. (1984),

$f_{\mathrm{V}}=3.58 \times 10^{-9} \times 10^{-0.4 m_{\mathrm{v}_{0}}} \mathrm{erg} \mathrm{cm}^{-2} \mathrm{~s}^{-1} \AA^{-1}$

with $m_{V_{0}}=m_{V}-2.175 c, c=1.47 E_{B-V}=0.096_{-0.022}^{+0.014}, m_{V}=$ $12.67 \pm 0.02, \log g=6.9 \pm 0.2$, and $M=0.530_{-0.015}^{+0.020} M_{\odot}$, the distance is derived from

$d=7.11 \times 10^{4} \sqrt{H_{v} \times M \times 10^{0.4 m_{v_{0}}-\log g}} \mathrm{pc}$.

With the Eddington flux at $\lambda_{\mathrm{eff}}=5454 \AA$ of our final model atmosphere, $H_{v}=(1.49 \pm 0.03) \times 10^{-3} \mathrm{erg} \mathrm{cm}^{-2} \mathrm{~s}^{-1} \mathrm{~Hz}^{-1}$, we derive a distance of $d=224_{-58}^{+46} \mathrm{pc}$. This is not in agreement with the distance ( $d=128.9 \mathrm{pc}$ ) of Harris et al. (2007). On the basis of the spectral analysis a value of $\log g=7.4$ which would be needed to reach agreement can be excluded (Sect. 3.3.2). 

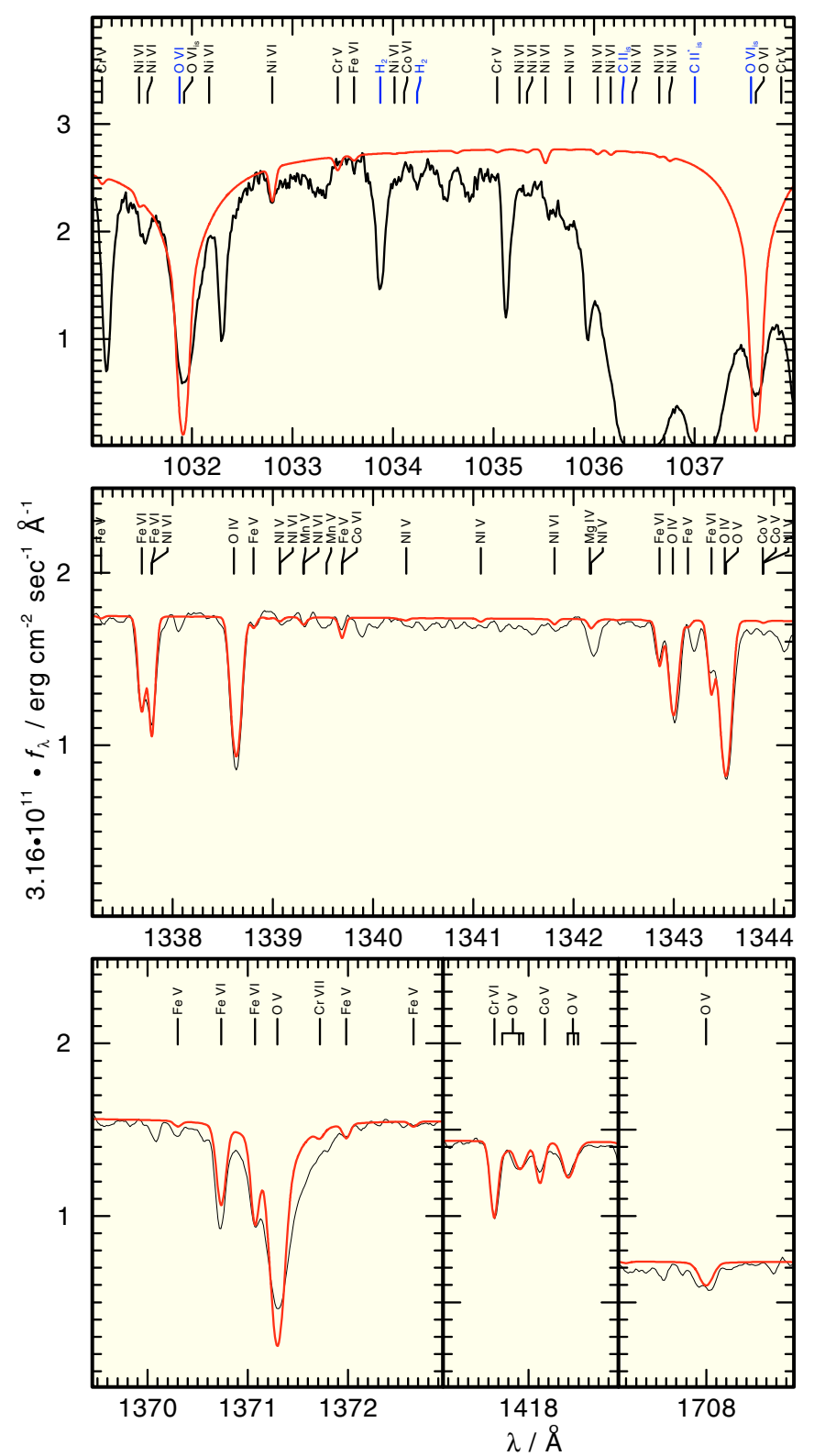

Fig. 13. Sections of the FUSE and STIS spectra of LS V $+46^{\circ} 21$ around O VI $\lambda \lambda 1031.9,1037.6 \AA$ (top), O IV $\lambda \lambda 1338.6,1343.0,1343.5 \AA$ (middle), $\mathrm{O} \vee \lambda 1371.3 \AA$ (bottom, left), O $\mathrm{V} \lambda \lambda 1417.7 .1417 .9,1418.4 \AA$ (bottom, middle), and $\mathrm{O} \vee \lambda 1708.0 \AA$ (bottom, right) compared with our final model.

\section{The FUSE spectrum and the ISM absorption model}

Along the line of sight to $\mathrm{LS} \mathrm{V}+46^{\circ} 21$ we detect interstellar absorption by $\mathrm{H}_{\mathrm{I}}, \mathrm{D}_{\mathrm{I}}, \mathrm{C}_{\mathrm{I}}, \mathrm{C}_{\mathrm{I}^{*}}, \mathrm{C}_{\mathrm{I}}{ }^{* *}, \mathrm{C}$ II, $\mathrm{C}_{\text {II }}^{*}, \mathrm{~N}_{\mathrm{I}}, \mathrm{N}_{\text {II }}, \mathrm{O}_{\mathrm{I}}, \mathrm{Al}$ II, Si II, Si III, P II, S I, S II, Cl I, Ar I, Fe II, Fe III, H $(J=0-9)$, HD $(J=0-1)$, and CO. Absorption by C IV $\lambda \lambda 1548,1550 \AA$, $\mathrm{N} v \lambda \lambda 1238,1242 \AA$, and $\operatorname{Si}$ IV $\lambda \lambda 1393,1402 \AA$ is also present at the velocity of the interstellar absorption of the species mentioned above $\left(v_{\text {helio }} \approx 6 \mathrm{~km} \mathrm{~s}^{-1}\right)$, in addition to the photospheric absorption at $\approx 20 \mathrm{~km} \mathrm{~s}^{-1}$.

At the FUSE and STIS resolutions all the interstellar absorption lines display only a single absorption component with a common velocity, $v_{\text {helio }} \approx 6 \mathrm{~km} \mathrm{~s}^{-1}$. However, the detection of several ionization stages for some of the species (e.g. C I, C II, and $\mathrm{C}$ IV) indicate that there must be at least three ISM

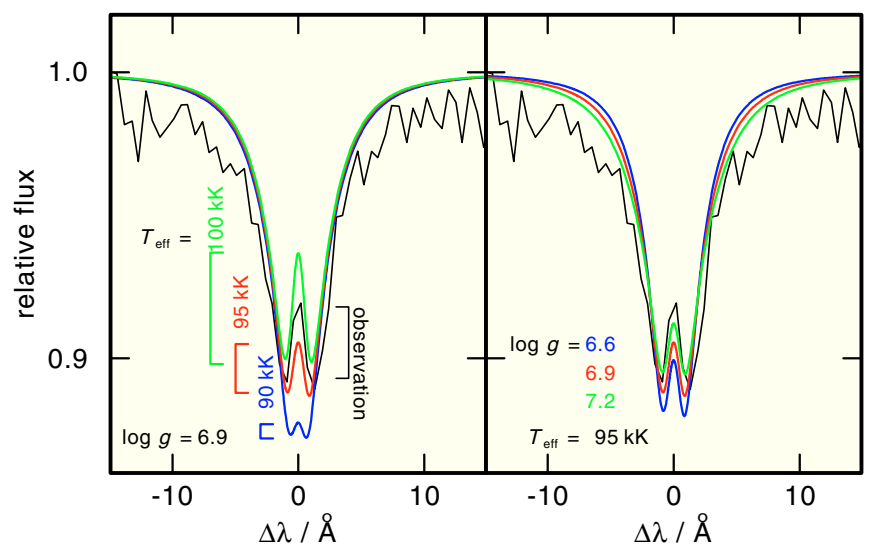

Fig. 14. Dependency of He II $\lambda 4686 \AA$ on $T_{\text {eff }}$ (left $)$ and $\log g$ (right). A section of the optical spectrum of LS V $+46^{\circ} 21$ around He II $\lambda 4686 \AA$ compared with theoretical line profiles calculated from models with $T_{\text {eff }}=90,95,100 \mathrm{kK}$ and $\log g=6.5,6.9,7.3$. In this parameter range, He II $\lambda 4686 \AA$ appears not very sensitive on $\log g$ but very sensitive on $T_{\text {eff }}$ in the line center. The brackets indicate the "strength" of the emission reversal in the line core.

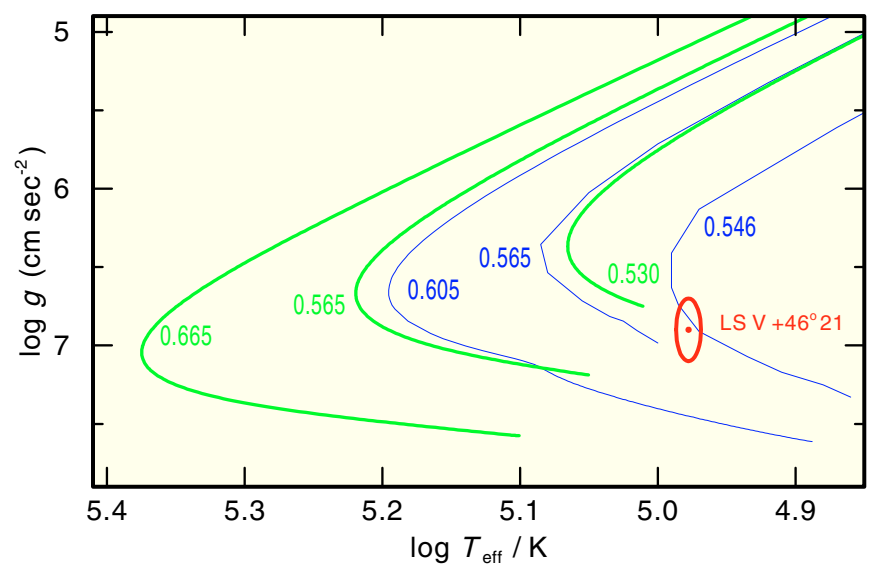

Fig. 15. Position (error ellipse) of LS V $+46^{\circ} 21$ in the $\log T_{\text {eff }}-\log g$ plane compared with evolutionary tracks of hydrogen-burning postAGB stars (Schönberner 1983; Blöcker \& Schönberner 1990, thin lines). The labels give the mass of the remnant in $M_{\odot}$. Note that a comparison with the new tracks by Althaus et al. (2005, thick lines) yields a lower remnant mass.

components along this line of sight: a cool component traced by molecular hydrogen and $\mathrm{C}_{\mathrm{I}}$ (amongst other species), a photoionized component traced by the high-ionization species ( $\mathrm{C}$ IV, $\mathrm{N} \mathrm{v}$, and Si IV), and a warm component where the bulk of the species such as $\mathrm{C}_{\text {II, }} \mathrm{N}_{\text {I }}, \mathrm{O}_{\text {I }}, \mathrm{S}_{\text {II }}, \mathrm{Fe}$ II, etc. resides. The highly ionized species likely reside in the photoionized gas associated with the PN where optical emission lines of $\mathrm{H} \alpha$ and [O III] have been detected (Fesen et al. 1981).

The analysis of the ISM along this line of sight is discussed by Oliveira (2007, in prep.).

With the procedure OWENS it is possible to model different interstellar absorption clouds with different chemical compositions, radial and turbulent velocities, temperature and column densities for each element included. We show two examples of the quality of our ISM modeling (Fig. 16).

The ISM fit is generally quite good, but incorporation of the new stellar model into the fits will help to refine the derived ISM parameters. 


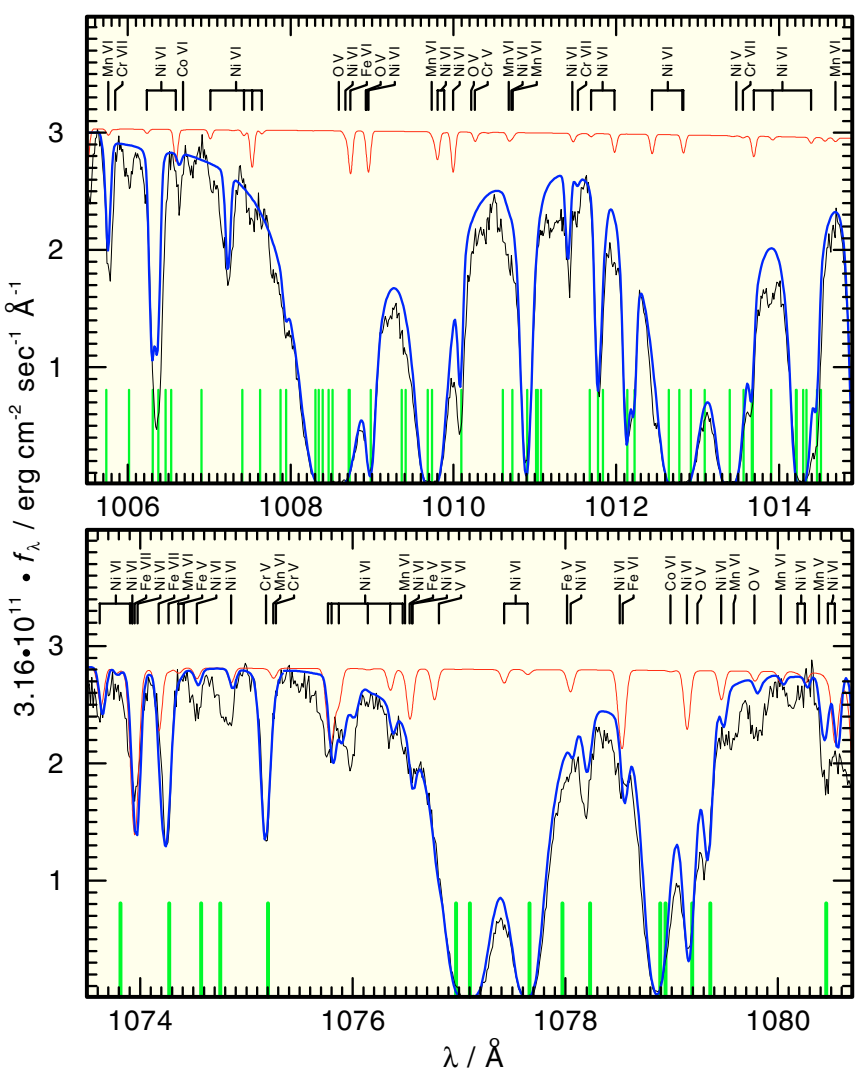

Fig. 16. Sections of the FUSE spectrum of LS V $+46^{\circ} 21$ compared with our best model of the ISM absorption (top panel, thick line) and with the combined ISM + model-atmosphere spectrum (bottom panel, thick line). The thin lines are the pure model-atmosphere spectrum. Most of the interstellar absorption is due to $\mathrm{H}_{2}$ (the line positions are marked by vertical bars at the bottom of the panels). The synthetic spectra are normalized to match the local continuum. Note that it is possible to identify a few isolated lines, e.g. Fe VII $\lambda 1073.9 \AA$, which are suitable for spectral analysis.

\section{The Galactic orbit}

We used the STIS spectrum of LS V $+46^{\circ} 21$ in order to determine its radial velocity out of a total of $54 \mathrm{Fe}$ VI and $\mathrm{Fe}$ VII photospheric lines. We obtained $v_{\text {rad }}=+20.6 \mathrm{~km} \mathrm{~s}^{-1}$ with a standard deviation of $\sigma_{v_{\text {rad }}}= \pm 1.5 \mathrm{~km} \mathrm{~s}^{-1}$. This is in agreement with $v_{\mathrm{rad}}=+22.4 \pm 3 \mathrm{~km} \mathrm{~s}^{-1}$ measured by Traulsen et al. (2005). Measurements from IUE spectra by Tweedy \& Napiwotzki (1992) and Holberg et al. (1998) (11.9 and $11.1 \mathrm{~km} \mathrm{~s}^{-1}$, respectively) may be less accurate because the star was possibly not well centered in the aperture (Holberg priv. comm.).

Kerber et al. (2004) have performed their orbit calculations with the radial velocity given by Tweedy \& Napiwotzki (1992) which is about a factor of two lower than measured from our STIS spectra. Thus, we have re-calculated the orbit (Fig. 17). We follow Pauli et al. (2003) and use the Galactic potential of Flynn et al. (1996) which includes a dark halo, bulge and stellar components, as well as three disks. The equations of motion are integrated using a fourth order Runge-Kutta scheme. To compute the tangential velocity we have used as a reference the proper motion of Kerber et al. (2004). Using the parallactic distance (128.9 pc) of Harris et al. (2007), we find that LS V $+46^{\circ} 21$ is a thin-disk object and its orbit extends about $\pm 0.20 \mathrm{kpc}$ perpendicular to the Galactic plane at a distance interval of $8.02 \mathrm{kpc}<\rho<8.85 \mathrm{kpc}$ from the Galactic center (Fig. 17). With our spectroscopic distance $(224 \mathrm{pc})$, there is not much difference: the orbit is now

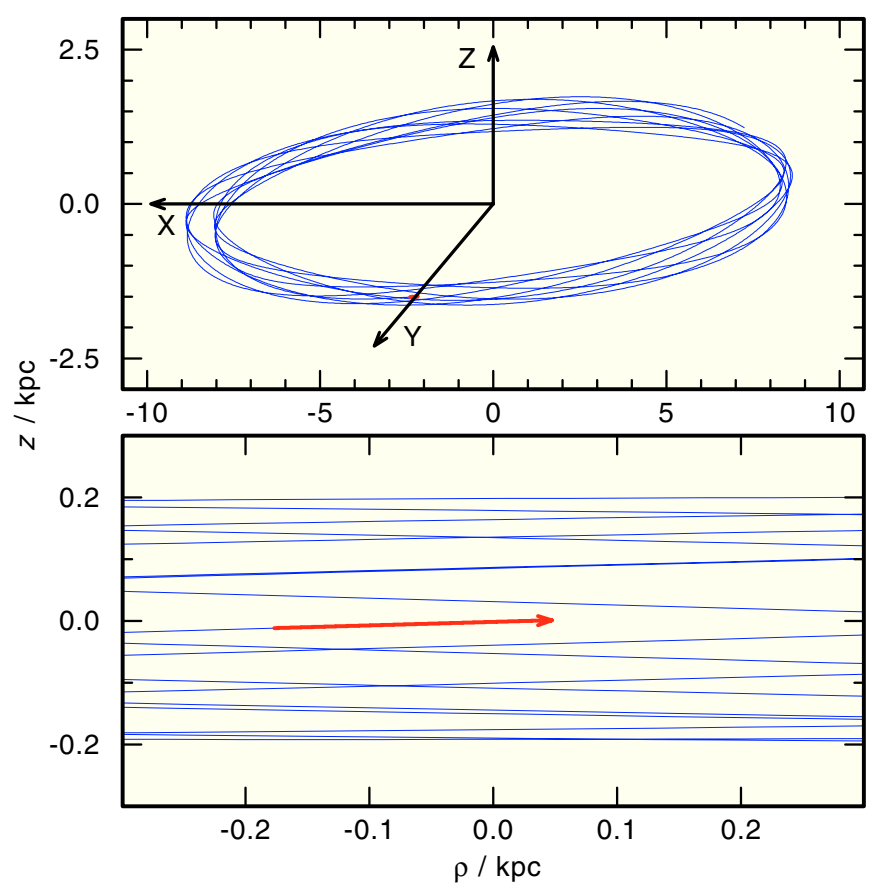

Fig. 17. The Galactic orbit of LS V $+46^{\circ} 21$ in the last 2 Gyrs in Galactocentric coordinates (top). At the bottom, a magnification around the present position of LS V $+46^{\circ} 21$ is shown. During its PN phase ( $\approx 460000$ years), $\mathrm{LS} \mathrm{V}+46^{\circ} 21$ has made its way along the (very small) thicker part of the track.

Table 4. Data related to the orbit of $\operatorname{LSV}+46^{\circ} 21 . z$ is the present height above the Galactic plane, the given angle is measured between the present orbit direction and the Galactic latitude.

\begin{tabular}{cccccccc}
\hline \hline$z$ & Angle & $U$ & $\sigma(U)$ & $V$ & $\sigma(V)$ & $W$ & $\sigma(W)$ \\
\cline { 2 - 7 } $\mathrm{pc}$ & $\circ$ & \multicolumn{6}{c}{$\mathrm{km} \mathrm{s}^{-1}$} \\
\hline 1 & 2 & -14.2 & 1.5 & 219.0 & 15.0 & 12.5 & 0.7 \\
\hline
\end{tabular}

confined between $\pm 0.25 \mathrm{kpc}$ from the Galactic plane, whereas it is lying at a distance $7.50 \mathrm{kpc}<\rho<8.90 \mathrm{kpc}$ from the center.

We have verified that this conserves total energy to better than $10^{-11}$, and the $z$ component of angular momentum to better than $10^{-10}$ for $L S V+46^{\circ} 21$.

The location of LS V $+46^{\circ} 21$ is presently about $1 \mathrm{pc}$ above the Galactic plane (Fig. 17). Its present velocity is summarized in Table 4, with $U$ in the Galactic disk, positive to the Galactic center, $V$ positive in the direction of Galactic rotation, and $W$ pointing to the North Galactic pole (NGP). We adopt the IAU convention (Kerr \& Lynden-Bell 1986) of $R_{\odot}=8.5 \mathrm{kpc}$ and $V_{R_{\odot}}=220 \mathrm{~km} \mathrm{~s}^{-1}$ for the Galactic rotational velocity at the Sun's position. For the solar peculiar motion we adopt the values of Dehnen \& Binney (1998) of $U_{\odot}=10.00 \mathrm{~km} \mathrm{~s}^{-1}, V_{\odot}=$ $5.25 \mathrm{~km} \mathrm{~s}^{-1}$, and $W_{\odot}=7.17 \mathrm{~km} \mathrm{~s}^{-1}$. The values of $U, V, W$, and of the Galacto-centric positions $X, Y, Z$, constitute the inputs to the code. Here, $X$ is positive in the direction of Galactic rotation, $Y$ positive from the Galactic center to the Sun, and $Z$ positive towards the NGP.

\section{Is Sh 2-216 a planetary nebula?}

LS V $+46^{\circ} 21$ is definitely a post-AGB star with a mass of $M=$ $0.550 M_{\odot}$ (Sect. 3.4). During its AGB mass-loss phase, it has lost about $75 \%$ of its initial mass into the ISM. Due to an interaction with the ambient ISM, the previously ejected envelope 
matter began to slow down about 45000 ago and $\mathrm{LS} \mathrm{V}+46^{\circ} 21$ is moving out of the geometric center. However, it is still surrounded by its "own" material and ionizes it. Narrow-band images of Sh 2-216 exhibit a shell-like nebula. Its expansion time can not be calculated reliably because the measured upper limit for the present $v_{\exp }$ of $4 \mathrm{~km} \mathrm{~s}^{-1}$ (Reynolds 1985) has a relatively large error range. Moreover, the low $v_{\exp }$ is the result of interaction with the ISM and, thus, the rate of change in that velocity over time is difficult to extrapolate back in time. If we assume a constant $v_{\exp }=4 \mathrm{~km} \mathrm{~s}^{-1}$, we get an upper limit for expansion time of about 460000 years. This expansion time is in agreement with evolutionary calculations of Schönberner (1983) for the stellar remnant. However, the real expansion time may be much smaller.

The classification of Sh 2-216 as a PN is corroborated but not proved, however, based on the latest parameters of LS V $+46^{\circ} 21$ and Sh 2-216 e.g. the determination of the ionized nebula mass could give evidence for Sh 2-216 being a "real" PN. We can calculate that, at a distance of 128.9 pc (Harris et al. 2007), an apparent diameter of $100^{\prime}$ gives a linear radius of $r_{\mathrm{sh}}=1.87 \mathrm{pc}$. If we assume that an old nebular shell has a thickness of $\approx 0.02 r_{\mathrm{sh}}$, assume a density of five particles $/ \mathrm{cm}^{3}$ (cf. Tweedy et al. 1995) and a solar composition $(\mu=1.26)$, then we arrive at $m_{\mathrm{sh}} \approx$ $0.2 M_{\odot}$. Although this may be a coarse approximation, the $m_{\mathrm{sh}}$ appears in agreement with an "average" PN mass.

\section{Conclusions}

The successful reproduction of the high-resolution FUSE and STIS spectra of LS V $+46^{\circ} 21$ by our synthetic spectra calculated from NLTE model atmospheres shows that - when done with sufficient care - theory works.

From the N IV - Nv, O Iv - O vi, Si IV - Si v, and $\mathrm{Fe} \mathrm{v}-\mathrm{Fe}$ vII ionization equilibria, we were able to determine $T_{\text {eff }}=95 \pm 2 \mathrm{kK}$ with - for these objects - unprecedented precision. Since this is a prerequisite for reliable abundance determinations (Table 5), the error limits for the determined abundances became also correspondingly small (Fig. 18).

The previously determined surface gravity of $\log g=6.9 \pm 0.2$ (Traulsen et al. 2005) is confirmed. Thus, the spectroscopic distance of $224 \mathrm{pc}$ (Sect.3.4) is too large compared with the parallax distance of 129 pc (Harris et al. 2007). It is disconcerting that we have such a large discrepancy for this star that is so well observed. $T_{\text {eff }}$ is well determined by different ionization equilibria and, thus, only a higher $\log g$ of about 7.4 (Sect. 3.3.2) could reconcile the discrepancy since the parallax distance requires a smaller stellar radius.

Napiwotzki (2001) investigated distance scales of old PN and found that trigonometric distances $\left(d_{\Pi}\right)$ are always smaller than spectroscopic distances determined by means of NLTE models $\left(d_{\text {NLTE }}\right)$. He found a distance ratio of $r_{\mathrm{obs}}=d_{\mathrm{NLTE}} / d_{\Pi}=$ $1.55 \pm 0.29$. However, simulations showed that a significant fraction of this discrepancy could be explained by biases caused by large relative errors of some parallax measurements (cf. Fig. 2 in Napiwotzki 2001). Recently, Harris et al. (2007) used improved error estimates for both distances and determined $r_{\mathrm{obs}}=1.3$. The parallax errors of several objects are significantly reduced and so are the biases as discussed in Harris et al. (2007).

In the case of $\mathrm{LS} \mathrm{V}+46^{\circ} 21$ we arrive at a ratio of $r_{\mathrm{obs}}=$ $1.74 \pm 0.35$ (cf. Napiwotzki 2001, $r_{\text {obs }}=1.46$ ) which is in accordance with the apparently general problem. However, one of the aims of this work was to solve this problem (cf. Napiwotzki 2001; Harris et al. 2007, and the discussions therein) - but has
Table 5. Photospheric abundances of LS V $+46^{\circ} 21$ normalized to $\log \sum_{i} \mu_{i} \varepsilon_{i}=12.15$, compared with solar abundances (Asplund et al. 2005).

\begin{tabular}{crr}
\hline \hline Element & \multicolumn{1}{c}{$\log \varepsilon$} & \multicolumn{1}{c}{$[\mathrm{x}]$} \\
\hline $\mathrm{H}$ & 12.035 & 0.112 \\
$\mathrm{He}$ & 10.634 & -0.818 \\
$\mathrm{C}$ & 8.880 & -0.510 \\
$\mathrm{~N}$ & 8.892 & 0.046 \\
$\mathrm{O}$ & 9.332 & -0.453 \\
$\mathrm{~F}$ & 5.875 & $<0.116$ \\
$\mathrm{Mg}$ & 8.889 & 0.053 \\
$\mathrm{Si}$ & 8.960 & 0.082 \\
$\mathrm{P}$ & 6.560 & -0.211 \\
$\mathrm{~S}$ & 8.265 & -0.301 \\
$\mathrm{Ar}$ & 7.781 & 0.079 \\
$\mathrm{Ca}$ & 8.583 & $<1.750$ \\
$\mathrm{Sc}$ & 5.442 & $<1.819$ \\
$\mathrm{Ti}$ & 7.320 & $<1.819$ \\
$\mathrm{~V}$ & 6.327 & $<1.699$ \\
$\mathrm{Cr}$ & 8.214 & 0.938 \\
$\mathrm{Mn}$ & 7.908 & 0.858 \\
$\mathrm{Fe}$ & 9.867 & 0.750 \\
$\mathrm{Co}$ & 7.468 & 0.858 \\
$\mathrm{Ni}$ & 8.638 & 0.719 \\
\hline
\end{tabular}

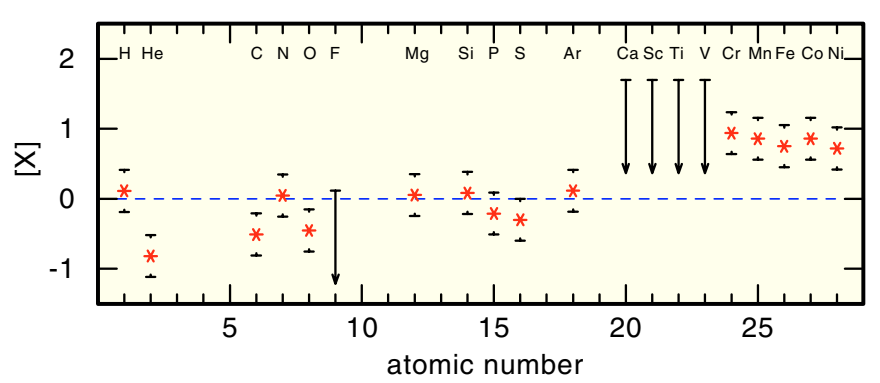

Fig. 18. Photospheric abundances of LS V $+46^{\circ} 21$ determined from detailed line profile fits. For F, Ca, Sc, Ti, and V upper limits can be found only.

remains at almost the same level although we use very elaborate NLTE model atmospheres. Since the TMAP NLTE model atmospheres (Sect. 3.1) used for this analysis are homogeneous, it would be highly desirable to calculate stratified models for LS V $+46^{\circ} 21$ in order to search for significant differences.

The derived abundance pattern (Fig. 18) gives evidence for an interplay of gravitational settling (e.g. the He and $\mathrm{C}$ abundances are strongly decreased by a factor of $\approx 0.15$ ) and radiative levitation (iron-group elements show an abundance up to $\approx 10 \times$ solar).

A comparison to abundance predictions (Fig. 19) from diffusion calculations of Chayer et al. (1995a) and Chayer et al. (1995b) shows a good agreement for C, N, O, Mg, S, and Fe with the DA values while we find that $\mathrm{Si}$ has a higher abundance and $\mathrm{Ar}$ and $\mathrm{Ca}$ have a lower abundance in our model.

Diffusion calculations for $\mathrm{He}$ in DAO white dwarfs have been presented by Vennes et al. (1988). These predict an upper limit of $\log \mathrm{He} / \mathrm{H} \approx-2.5$ for a $0.55 M_{\odot}$ and $T_{\text {eff }}=95 \mathrm{kK}$ star. We arrive at $\log \mathrm{He} / \mathrm{H}=-2.0$ in our final model.

New diffusion calculations which include all elements from hydrogen to nickel could further improve our models, e.g. provide abundance predictions for those species which are not identified in the spectrum of LS V $+46^{\circ} 21$.

Good et al. (2005) have determined C, N, O, Si, and Fe abundances using FUSE observations of 16 DAO white dwarfs. They 


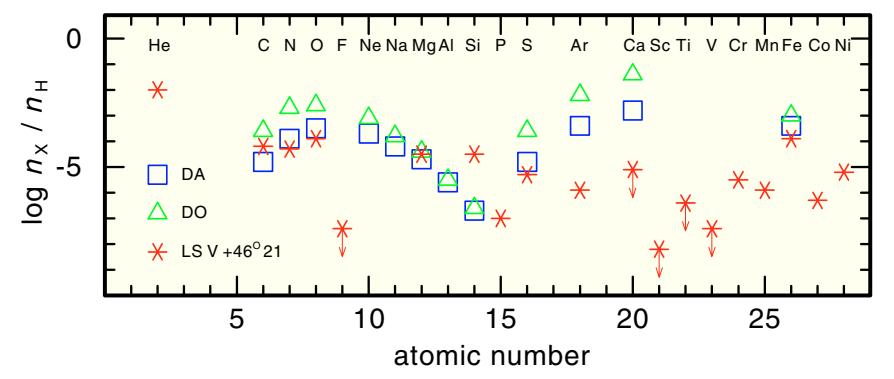

Fig. 19. Comparison of the elemental number ratios found in our spectral analysis compared to predictions of diffusion calculations for DA and DO models (Chayer et al. 1995b,a) with $T_{\text {eff }}=95 \mathrm{kK}$ and $\log g=7$.

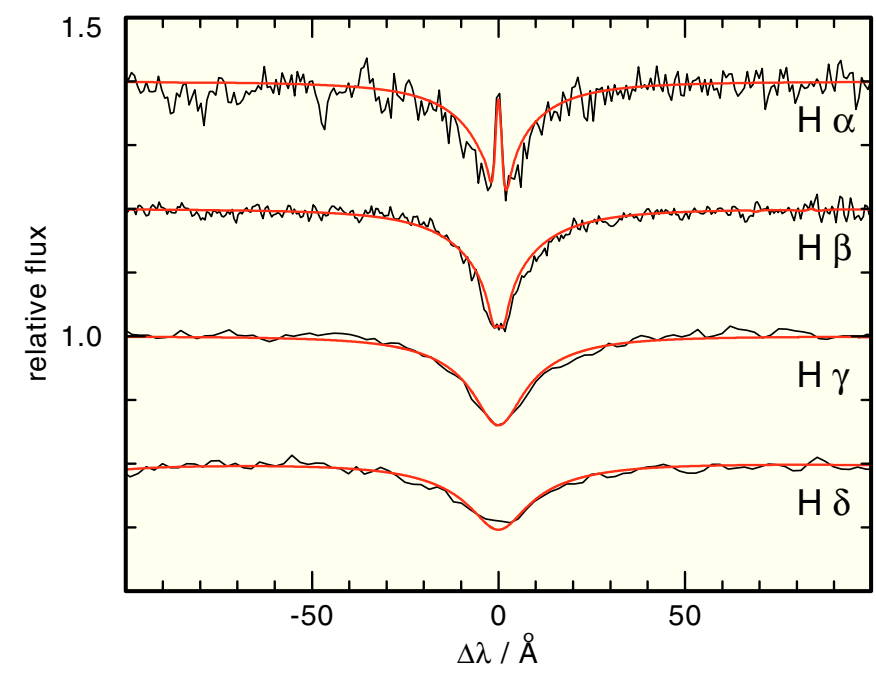

Fig. 20. Synthetic Balmer-line spectra of our final model $\left(T_{\text {eff }}=95 \mathrm{kK}\right.$, $\log g=6.9$ ) compared with the observation (cf. Napiwotzki 1999).

compare their results with values given by Chayer et al. (1995a) and find deviations of $+0.25,-0.50,-0.10,+3.0$, and $+0.1 \mathrm{dex}$ (interpolated by us in their figures at $T_{\text {eff }}=95 \mathrm{kK}$ ), respectively. This is within 0.5 dex in agreement with our result.

In our metal-line blanketed NLTE model atmospheres which include the opacity of 20 species from $\mathrm{H}$ to Ni (Sect. 3), the BLP (Sect. 1) vanishes (Fig. 20) if the synthetic spectra are compared to the available medium-resolution optical spectra at $S / N \approx 30$. High-resolution and high- $S / N$ spectra are highly desirable to check for remaining discrepancies.

The reddening of $E_{B-V}=0.065_{-0.015}^{+0.010}$ towards LS V $+46^{\circ} 21$ is much higher than expected from the Galactic reddening law (Sect. 2.3) possibly because of additional reddening due to dust in the nebula Sh 2-216.

\section{Future work: atomic and line-broadening data}

With the analysis of the high-resolution, high- $S$ / $N$ UV spectrum of LS V $+46^{\circ} 21$ it is demonstrated that state-of-the-art NLTE spectral analysis has presently arrived at a high level of sophistication. The main limitation now encountered is the lack of reliable atomic and line-broadening data.

Going to higher resolution and $S / N$ in the observations reveals uncertainties in atomic data even for the most abundant species. Thus, it is a challenge for atomic physics to provide properly measured atomic data (for a wide range of ionization stages).

Discrepancies between our synthetic line profiles of the resonance doublets of $\mathrm{N}$ v, O VI, and S VI and the observation may have their reason in insufficient line-broadening tables. Even for strategic lines like, e.g., $\mathrm{O} \vee \lambda 1371 \AA$ which is often used to determine $T_{\text {eff }}$, no appropriate tables are available. Theoretical physics should provide data which covers the astrophysical relevant temperature and density space.

Better atomic and line-broadening data will then strongly improve future spectral analyses and thus, make determinations of photospheric properties more reliable.

Acknowledgements. We like to thank Hugh Harris and Ralf Napiwotzki for comments and discussion. T.R. is supported by the German Astrophysical Virtual Observatory project of the German Federal Ministry of Education and Research (BMBF) under grant 05 AC6VTB. J.W.K. is supported by the FUSE project, funded by NASA contract NAS532985. This research has made use of the SIMBAD Astronomical Database, operated at CDS, Strasbourg, France. This work has been done using the profile fitting procedure OwENs developed by $\mathrm{M}$. Lemoine and the FUSE French Team.

\section{References}

Althaus, L. G., Miller Bertolami, M. M., Córsico, A. H., García-Berro, E., \& Gil-Pons, P. 2005, A\&A, 400, L 1

Asplund, M., Grevesse, N., \& Sauval, A. J. 2005, in: Cosmic Abundances as Records of Stellar Evolution and Nucleosynthesis, ed. T. G. Barnes III, \& F. N. Bash, ASP Conf. Ser., 336, 25

Barstow, M. A., Hubeny, I., Holberg, J. B. 1998, MNRAS, 299, 520

Bergeron, P., Ruiz, M.-R., \& Leggett, S. K. 1993, ApJ, 407, L85

Bergeron, P., Wesemael, F., Beauchamp, A., et al. 1994, ApJ, 432, 305

Blöcker, T., \& Schönberner, D. 1990, A\&A, 240, L1

Borkowski, K. J., Sarazin, C. L., \& Soker, N. 1990, ApJ, 360, 173

Chayer, P., Fontaine, G., \& Wesemael, F. 1995a, ApJS, 99, 189

Chayer, P., Vennes, S., Pradhan, A. K., et al. 1995b, ApJ, 454, 429

Cheselka, M., Holberg, J. B., Watkins, R., Collins, J., \& Tweedy, R. W. 1993, AJ, 106, 2365

Cudworth, K., \& Reynold, R. J. 1985, PASP, 97, 175

Dehnen, W., \& Binney, J. J. 1998, MNRAS, 298, 387

Dixon, W. V., et al. 2007, PASP, in press

Dimitrijević, M. S., \& Sahal-Bréchot, S. 1992a, A\&AS, 93, 359

Dimitrijević, M. S., \& Sahal-Bréchot, S. 1992b, A\&AS, 95, 109

Dimitrijević, M. S., \& Sahal-Bréchot, S. 1993, A\&AS, 100, 91

Dimitrijević, M. S., Sahal-Bréchot, S., \& Bommier, V. 1991, A\&AS, 89, 581

Feibelman, W. A., \& Bruhweiler, F. C. 1990, ApJ, 357, 548

Fesen, R. A., Blair, W. P., \& Gull, T. R. 1981, ApJ, 245, 131

Flynn, C., Sommer-Larsen, J., \& Christensen, P. R. 1996, MNRAS, 281, 1027

Good, S. A., Barstow, M. A., Burleigh, M. R., et al. 2005, MNRAS, 363, 183

Groenewegen, M. A. T., \& Lamers, H. J. G. L. M. 1989, A\&AS, 79, 359

Hardorp, J., Theile, I., \& Voigt, H. H. 1965, in: Luminous Stars in the Northern

Milky Way, Vol. 5, Hamburger Sternwarte, Warner \& Swasey Obs., 5

Harris, H. C., Dahn, C. C., Canzian, B., et al. 2007, AJ, 133, 631

Heber, U., Hunger, K., Jonas, G., \& Kudritzki, R.-P. 1984, A\&A, 130, 119

Hoffmann, A. I. D. 2005, diploma thesis, Universität Tübingen, Germany

Holberg, J. B., Barstow, M. A., \& Sion, E. M. 1998, ApJS, 119, 207

Hummer, D. G., Berrington, K. A., Eissner, W., et al. 1993, A\&A, 279, 298

Jahn, D., Rauch, T., Reiff, E., et al. 2007, A\&A, 466, 281

Kerber, F., Mignani, R. P., Pauli, E.-M., Wicenec, A., \& Guglielmetti, F. 2004, A\&A, 420, 207

Kerr, F. J., \& Lynden-Bell, D. 1986, MNRAS, 221, 1023

Kruk, J. W., \& Werner, K. 1998, ApJ, 502, 858

Kurucz, R. L. 1996, in: Stellar surface structure, ed. K. G. Strassmeier, \& J. L. Linsky (Dordrecht: Kluwer), IAU Symp., 176, 523

Lemke, M. 1997, A\&AS, 122, 285

McCook, G. P., \& Sion, E. M. 1999, ApJS, 121, 1

Moos, H. W., Cash, W. C., Cowie, L. L., et al. 2000, ApJ, 538, L1

Napiwotzki, R. 1992, Lecture Notes in Physics, Vol. 401, Springer-Verlag, Berlin, 310

Napiwotzki, R. 1993, Acta Astron., 43, 343

Napiwotzki, R. 1999, A\&A, 350, 101

Napiwotzki, R. 2001, A\&A, 367, 973

Napiwotzki, R., \& Schönberner, D. 1993, in: White Dwarfs: Advances in Observation and Theory, ed. M\&. A\&. Barstow NATO Advanced Science Institutes (ASI) Series C (Dordrecht: Kluwer), 403, 99

Napiwotzki, R., \& Rauch, T. 1994, A\&A, 285, 603 
Noerdlinger, P. D. \& Dynan S. E. 1975, ApJS, 29, 185

Pauli, E.-M., Napiwotzki, R., Altmann, M., et al. 2003, A\&A, 400, 877

Rauch, T. 1993, A\&A, 276, 171

Rauch, T. 1997, A\&A, 320, 237

Rauch, T. 2003, A\&A, 403, 709

Rauch, T., \& Deetjen, J. L. 2003, in Stellar Atmosphere Modeling, ed. I. Hubeny, D. Mihalas, \& K. Werner, ASP Conf. Ser., 288, 103

Rauch, T., Köppen, J., \& Werner, K. 1996, A\&A, 310, 613

Rauch, T., Kerber, K., \& Pauli, E.-M. 2004, A\&A, 417, 647

Reynolds, R. J. 1985, ApJ, 288, 622

Sahnow, D. J., Moos, H. W., Ake, T. B., et al. 2000, ApJ, 538, L7

Savitzky, A., \& Golay, M. J. E. 1964, Analytical Chemistry 36, 1627

Schönberner, D. 1983, ApJ, 272, 708

Schöning, T., \& Butler, K. 1989, A\&AS, 78, 51

Seaton, M. J. 1979, MNRAS, 187, 73

Seaton, M. J., Yan, Y., Mihalas, D., \& Pradhan, A. K. 1994, MNRAS, 266, 805
Sharpless, S. 1959, ApJS, 4, 257

Sofia, U. J., Wolff, M. J., Rachford, B., et al. 2005, ApJ, 625, 167

Traulsen, I., Hoffmann, A. I. D., \& Rauch, T. 2005, in White Dwarfs, ed. D. Koester, \& S. Moehler, ASP Conf. Ser., 344, 325

Tweedy, R. W., \& Napiwotzki, R. 1992, MNRAS, 259, 315

Tweedy, R. W., Martos, M. A., \& Noriega-Crespo, A. 1995, ApJ, 447, 257

Vennes, S., Pelletier, C., Fontaine, G., \& Wesemael, F. 1988, ApJ, 331, 876

Werner, K. 1986, A\&A, 161, 177

Werner, K. 1996, ApJ, 457, L39

Werner, K., Deetjen, J. L., Dreizler, S., Rauch, T., \& Kruk, J. W. 2003a, in Planetary Nebulae: Their Evolution and Role in the Universe, ed. S. Kwok, M. Dopita, \& R. Sutherland, Proc. IAU Symp., 209, 169

Werner, K., Dreizler, S., Deetjen, J. L., et al. 2003b, in Stellar Atmosphere Modeling, ed. I. Hubeny, D. Mihalas, \& K. Werner, ASP Conf. Ser., 288, 31

Werner, K., Rauch, T., \& Kruk, J. W. 2005, A\&A, 433, 641 
T. Rauch et al.: High-resolution FUSE and HST ultraviolet spectroscopy of the white dwarf central star of Sh 2-216, Online Material p 1

\section{Online Material}


T. Rauch et al.: High-resolution FUSE and HST ultraviolet spectroscopy of the white dwarf central star of Sh 2-216, Online Material p 2

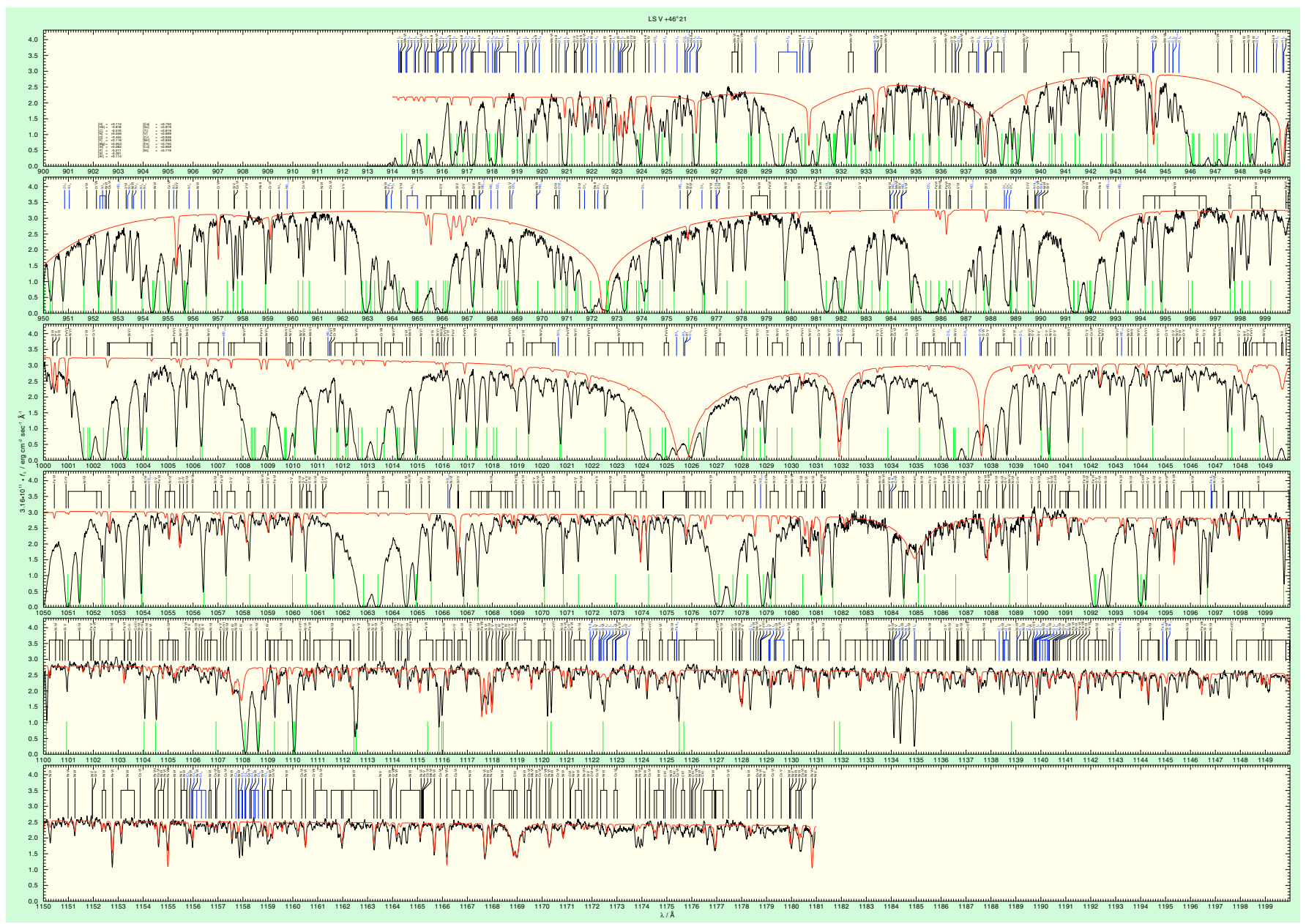

Fig. A.1. 
T. Rauch et al.: High-resolution FUSE and HST ultraviolet spectroscopy of the white dwarf central star of Sh 2-216, Online Material p 3

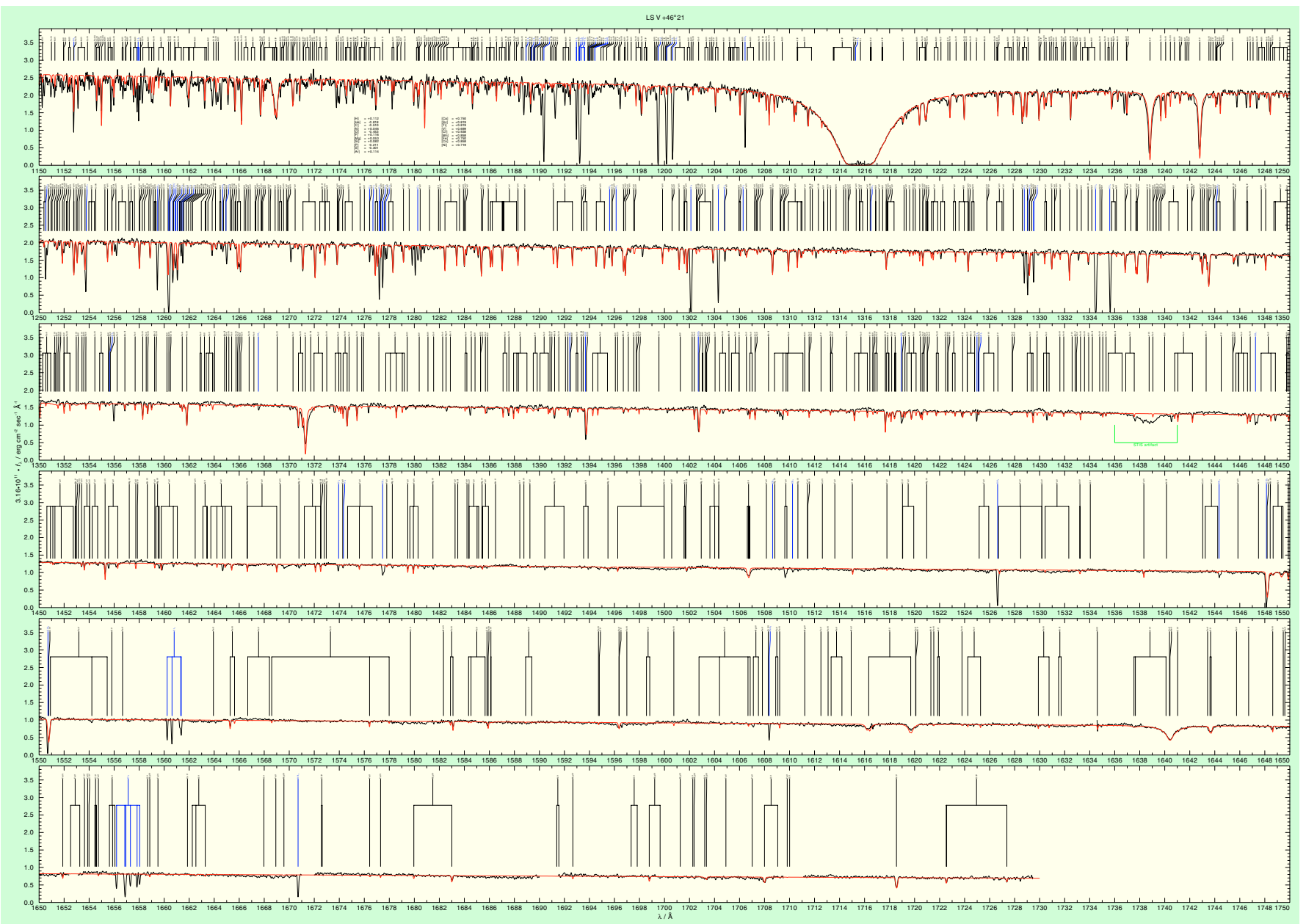

Fig. A.2. 